\title{
A Systematic Approach to Plant-Wide Control Based On Thermodynamics
}

\author{
Luis T. Antelo, Irene Otero-Muras, Julio R. Banga, \\ Antonio A. Alonso * \\ Process Engineering Group,Instituto de Investigaciones Marinas-CSIC \\ C/Eduardo Cabello, 6 - 36208 Vigo, Spain
}

\begin{abstract}
In this work, a systematic approach to plant-wide control design is proposed. The method combines ingredients from process networks, thermodynamics and systems theory to derive robust decentralized controllers that will ensure complete plant stability. As a first step, the considered process system is decomposed into abstract mass and energy inventory networks. In this framework, conceptual inventory control loops are then designed for the mass and energy layers to guarantee that the states of the plant, both in terms of extensive and intensive properties, will converge to a compact convex region defined by constant inventories. This result by itself does not ensure the convergence of intensive variables to a desired operation point as complex dynamic phenomena such as multiplicities may appear in the invariant set. In order to avoid these phenomena, thermodynamics naturally provides the designer, in these convex regions, with a legitimate storage or Lyapunov function candidate, the entropy, that can be employed to ensure global stability. Based on this, the control structure design procedure is completed with the realization of the conceptual inventory and intensive variable control loops over the available degrees of freedom in the system. To that purpose, both PI and feedback linearization control are employed. The different aspects of the proposed methodology will be illustrated on a non-isothermal chemical reaction network.
\end{abstract}

Key words: Plant-wide Control, Process Networks, Irreversible Thermodynamics, Inventory Control.

* Corresponding author
Email address: antonio@iim.csic.es (Antonio A. Alonso).

Preprint submitted to Elsevier Science

11 September 2006 


\section{Introduction}

Over the years, the area of plant-wide control has attracted the process engineering community as a challenging problem which drives continuing research efforts. By re-phrasing Professor S. Skogestad words (Larsson and Skogestad, 2000): The objective of plant-wide control is not the tuning of a given set of control loops on a chemical plant but rather the control philosophy of the overall plant with emphasis on the structural decisions. A number of solutions to it were suggested, lying in between the following two extremes:

(1) A hierarchical decomposition of the original control design problem based on heuristic rules. The heuristic logic is developed so to keep process variability and therefore the operational plant objectives under acceptable limits for a given set of disturbances (see Buckley, 1964; Luyben et al., 1997 or Skogestad, 2002, for further information about this decomposition)

(2) A mathematically oriented approach based on the solution of a given large scale mixed integer nonlinear programming dynamic optimization problem, which in the limit should be able to simultaneously determine the optimal process units size and their interconnections as well as the optimal control scheme configuration (see Biegler and Grossman, 2004, for an excellent review).

Unfortunately, both lines of attack are hampered by a number of drawbacks which prevent their systematic application to general classes of process plants: On the one hand, the hierarchical approach usually leads to conflicting decisions only unravelled on a case by case basis. On the other hand, the mathematically oriented approach is limited by the high dimensionality of the problem, the nonlinear character of the constitutive equations and the restrictions imposed by the definition of the objective function. In addition, the stability and robustness of the resulting control scheme is highly dependent on the number, type and characteristics of plant disturbances. In fact, such disturbances usually need to be known beforehand as part of the control design problem. Furthermore, nothing prevents the resulting control configuration of exhibiting instabilities under a different class of disturbances.

To overcome these issues, we combine previous results that link thermodynamics with passivity and Lyapunov theory. The basic ingredients of the theory have been established by Alonso and Ydstie (1996) and Ydstie and Alonso (1997) in the context of passive control design and control of distributed systems (Alonso and Ydstie, 2001), and transport reaction systems (Ruszkowski et al., 2005). A similar line of arguments was employed by Farschman et al. (1998) to derive mass and energy inventory control concepts. Hangos et al. 
(1999) applied them to define structural stability conditions for separation process networks. Thermodynamics was also central in the work by Bao et al. (2002) to design passivity-based decentralized control of failure-tolerant systems.

In this paper, our aim is to apply and extend these results in order to systematically design stable decentralized control structures for process plants. The proposed approach leads to a hierarchical decentralized inventory control structure which simultaneously ensures convergence of mass and energy inventories to a compact convex set in which the system will remain on.

It must be pointed out that this convex region definition tries to generalize the approach by Hangos et al. (1999), where extensive variables are assumed constant, to derive the structural stability conditions. Once in this region, convergence of the intensive variables (temperatures, pressures and concentrations) to a unique stable steady-state can be enforced by a number of well established control schemes (for instance, PI control, feedback linearization controllers, etc.).

Finally, it must be remarked that our approach complements others such as the one recently proposed in the context of chemical reactors (Gonzalez and Alvarez, 2005) since it provides the designer with the required physical insight and systematic tools to select inputs and outputs, decide what variables to estimate, and select stabilizing control alternatives.

The present paper is structured as follows: In Section 2, a formal representation of chemical plants in terms of interconnected mass and energy networks is presented as a first step in designing control structures. In this Section, we formally introduce the so-called inventory network and describe the general algebraic structure underlying its dynamics. The thermodynamic formalism and its application in designing conceptual inventory controllers are presented in Section 3. The realization of these control loops over the real available degrees of freedom of the process and the intensive control problem, as the final steps in the control design, are also developed in this Section. Finally, in Section 4 the approach is applied to design a decentralized control structure for a non-isothermal reactor network.

\section{The Underlying Structure of Process Networks}

As a first step in the control structure design procedure, we systematize the process representation by making use of the ideas of process networks as a graph representation of the process flowsheet. A process network is defined by a number $j=1, \ldots, \theta$ of well mixed homogeneous material regions connected 
by material and energy fluxes we will refer to as nodes, plus an extra region $j=0$ which represents the environment. To each node $j$ in the network, we associate a state vector $z_{j} \in \mathbb{R}^{c+1}$ of the form:

$$
z_{j}=\left(n_{j}^{1}, \ldots, n_{j}^{c}, u_{j}\right)^{T}
$$

where $n_{j}^{k}$ represents the mole number of component $k, u_{j}$ is the internal energy and $c$ stands for the total number of chemical species. Graphically, each phase present in the process is represented by one circle denoting a node, and solid circles symbolize the environment. Nodes and environment are connected by a set of $\theta$ convective fluxes which, for every node, we refer to as $f_{j} \in \mathbb{R}^{+c}$ and $p_{j}\left(f_{j}\right) \in \mathbb{R}^{+}$for component and energy, respectively. In addition, we have the following relationship between mass and energy convective flows:

$$
p_{j}\left(f_{j}\right)=\sum_{k=1}^{c} u_{j}^{k} f_{j}^{k}
$$

with $u_{j}^{k}$ being the energy density associated to component $k$ in node $j$. Since

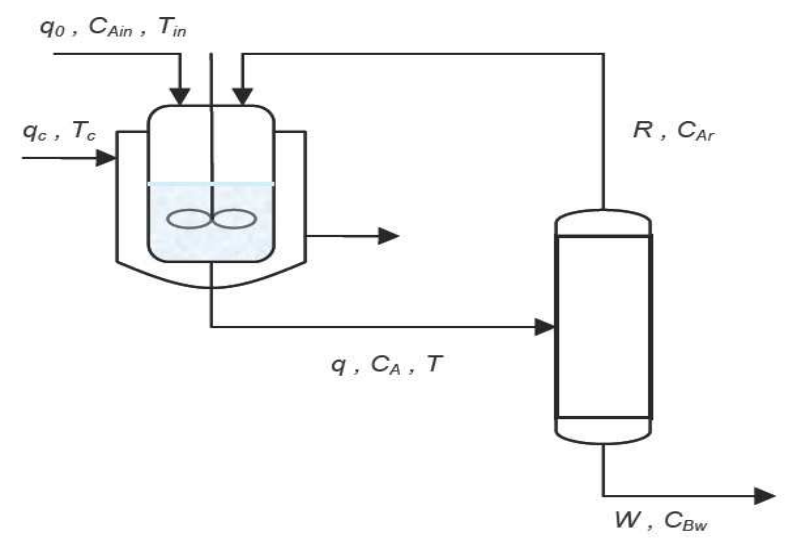

Fig. 1. Reactor-Separator process flowsheet (See notation list in Table 4.1). $R, W$, $C_{A_{r}}$ and $C_{B_{w}}$ represent the flowrates and concentrations of $A$ and $B$ in the recycle and final product streams, respectively.

energy is transported by convective flows, we also have that $p_{j}(0)=0$. In the graphical representation, these mass and energy convective fluxes are denoted by solid and dashed arrows, respectively. Nodes in the network can also be interconnected by dissipative transfer fluxes collected in vectors $\varphi^{k} \in \mathbb{R}^{+d_{c}}$ (with $k=1 \ldots, c$ ) and $\psi \in \mathbb{R}^{+d_{u}}$, where $d_{c}$ and $d_{u}$ stand for mass and energy dissipative transfer, respectively. In the network graphs, these dissipative fluxes are represented by solid and dashed double-head arrows for mass and energy, respectively. In order to exemplify this process network formalism, let us consider the system depicted in Figure 1. This process includes a jacketed exothermic reactor, where reactant $A$ is transformed to product $B$ through the first order exothermic reaction $A \rightarrow B$, plus a flash unit where $B$ is obtained as liquid product and reactant $A$ is recycled. 
The process flowsheet in Figure 1 can be represented as the process network depicted in Figure 2, where the reactor and its jacket are denoted by nodes 1 and 2, respectively, interconnected through a dissipative energy flow $\psi$. Both nodes are also connected with the environment (node 0 is represented by small solid nodes) by convective flows (a mass convective flow $-f_{0_{1}}$ - for the reactor case and an energy flow $-p_{0_{2}}$ - for the jacket node). Nodes 1 and 3 are connected through a convective flow $\left(f_{1}, p_{1}\right)$. Note that nodes 3 and 4 denote the liquid and vapor phases in the flash unit, respectively. It must be pointed out that the formalism, as it stands, can also accommodate multiple connections between nodes. This can be done as suggested in Hangos et al. (1999), by defining the fraction $\alpha_{i j}$ of flow in the direction from node $i$ to node $j$ so that $\sum_{i} \alpha_{i j}=1$. For the case shown in Figure 2, part of the flow leaving node $4\left(f_{4}, p_{4}\right)$ is recycled to node $1\left(\alpha_{41}\right)$, while the remaining goes to node $0\left(1-\alpha_{41}=\alpha_{40}\right)$. Finally, the product stream is denoted as the convective flow leaving node 3 to node $0\left(f_{3}, p_{3}\right)$. In summary, for this particular network, we have that $n=4$ (number of nodes), $c=2$ (molecular species, $\mathrm{A}$ and $\mathrm{B}$ ), $d_{c}=1$ (number of mass dissipative connections) and $d_{u}=1$ (number of heat transfer connections).

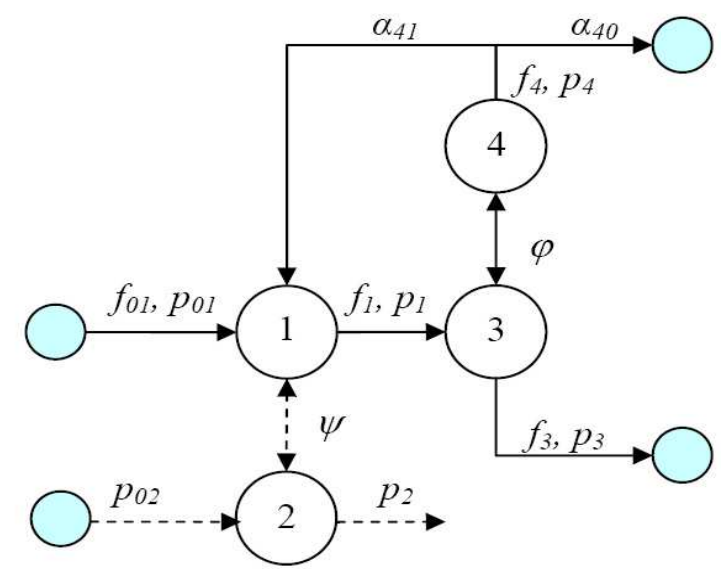

Fig. 2. Process network of the reactor-separator process.

Network dynamics obey standard conservation principles for mole number and energy which, with some abuse of notation, can be formally stated as:

$$
\begin{gathered}
\dot{n}^{k}=N_{0} f_{0}^{k}+N_{\phi} f^{k}+N_{\varphi} \varphi^{k}+\gamma W \quad n^{k}, f^{k} \in \mathbb{R}^{+\theta} ; f_{0}^{k} \in \mathbb{R}^{+d_{i}} ; k=1, \ldots, c ; \\
\dot{u}=N_{0} p_{0}+N_{\phi} p+N_{\psi} \psi+Q \quad u, p \in \mathbb{R}^{+\theta} ; p_{0} \in \mathbb{R}^{+d_{i}}
\end{gathered}
$$

with $f_{0} \in \mathbb{R}^{d_{i}}$ being the vector of external convective inputs (and $d_{i}$ the number of inputs), and matrices $N_{0} \in \mathbb{R}^{+\theta \times d_{i}}, N_{\phi} \in \mathbb{R}^{\theta \times \theta}, N_{\varphi} \in \mathbb{R}^{\theta \times d_{c}}$ and $N_{\psi} \in \mathbb{R}^{\theta \times d_{u}}$ describing dissipative and convective network interconnections. Finally, the extra-terms $\epsilon W$ and $Q$ in Eqns. (3)-(4) have been included to account, when necessary, either for chemical reaction units or external heat sources. For the reaction term, $\epsilon$ and $W$ are the stoichiometric and reaction rate vectors, respectively. 
For the process network depicted in Figure 2, since neither component $A$ nor $B$ are present in node 2 and the reaction takes place only on node 1 , the material network dynamics (Eqn. 3) becomes:

$$
\left[\begin{array}{c}
\dot{n}_{1}^{k} \\
\dot{n}_{3}^{k} \\
\dot{n}_{4}^{k}
\end{array}\right]=\left[\begin{array}{ccc}
-1 & 0 & \alpha_{41} \\
1 & -1 & 0 \\
0 & 0 & -1
\end{array}\right]\left[\begin{array}{c}
f_{1}^{k} \\
f_{3}^{k} \\
f_{4}^{k}
\end{array}\right]+\left[\begin{array}{c}
1 \\
0 \\
0
\end{array}\right] f_{01}^{k}+\left[\begin{array}{c}
0 \\
-1 \\
1
\end{array}\right] \varphi^{k}+\left[\begin{array}{cc}
\epsilon_{A} & \epsilon_{B} \\
0 & 0 \\
0 & 0
\end{array}\right]\left[\begin{array}{c}
W_{1} \\
W_{2}
\end{array}\right]
$$

Note that the signs of the stoichiometric coefficients $\epsilon_{A}$ and $\epsilon_{B}$ will depend on whether one considers the reactant (positive) or the product (negative).

The corresponding energy network (Eqn. 4) is of the form:

$$
\left[\begin{array}{c}
\dot{u}_{1} \\
\dot{u}_{2} \\
\dot{u}_{3} \\
\dot{u}_{4}
\end{array}\right]=\left[\begin{array}{cccc}
-1 & 0 & 0 & \alpha_{41} \\
0 & -1 & 0 & 0 \\
1 & 0 & -1 & 0 \\
0 & 0 & 0 & -1
\end{array}\right]\left[\begin{array}{l}
p_{1} \\
p_{2} \\
p_{3} \\
p_{4}
\end{array}\right]+\left[\begin{array}{ll}
1 & 0 \\
0 & 1 \\
0 & 0 \\
0 & 0
\end{array}\right]\left[\begin{array}{l}
p_{0_{1}} \\
p_{0_{2}}
\end{array}\right]+\left[\begin{array}{c}
1 \\
-1 \\
0 \\
0
\end{array}\right] \psi
$$

Let us now define a dissipative sub-network $\mathcal{D}\left(\theta_{j}\right)$ as that constituted by the collection of $\theta_{j}$ nodes only interconnected through dissipative fluxes. Then, any process network can be viewed as the convective interconnection of $\ell$ dissipative sub-networks, so that $\theta=\sum_{j=1}^{\ell} \theta_{j}$. Each dissipative sub-network $\mathcal{D}$ has a given component and total inventory defined as:

$$
n_{\mathcal{D}}^{k}=\sum_{i \in \mathcal{D}} n_{i}^{k} \quad n_{\mathcal{D}}=\sum_{k=1}^{c} n_{\mathcal{D}}^{k}
$$

We also introduce $\forall i \in \mathcal{D}$ the following fluxes:

$$
F_{i}=\sum_{k=1}^{c} f_{i}^{k} \quad F_{\mathcal{D}}=\sum_{i \in \mathcal{D}} F_{i}
$$

The dissipative sub-network concept, we just defined, allows us to consider that any process network has an associated inventory network, which formally can be constructed by projecting Eqns. (3)-(4) onto a set of linear operators $P_{\varphi} \in \mathbb{R}^{\ell \times \theta}$ and $P_{\psi} \in \mathbb{R}^{\ell \times \theta}$ satisfying $P_{\varphi} N_{\varphi}=0$ and $P_{\psi} N_{\psi}=0$. By defining the vectors of inventories and fluxes $n_{\mathcal{I}}, u_{\mathcal{I}}, F_{\mathcal{I}}, p_{\mathcal{I}} \in \mathbb{R}^{+\ell}$, the following inventory representation is obtained:

$$
\begin{array}{cc}
\dot{n}_{\mathcal{I}}=\mathcal{N} F_{\mathcal{I}}+\mathcal{R} & n_{\mathcal{I}}, F_{\mathcal{I}}, \mathcal{R} \in \mathbb{R}^{+\ell} \\
\dot{u}_{\mathcal{I}}=\mathcal{N} p_{\mathcal{I}} & u_{\mathcal{I}}, p_{\mathcal{I}} \in \mathbb{R}^{+\ell}
\end{array}
$$


where $\mathcal{R}=\sum_{k=1}^{c} P_{\varphi} \nu W$ and $\mathcal{N} \in \mathbb{R}^{\ell \times \ell}$ satisfies:

$$
\mathcal{N} F_{\mathcal{I}} \equiv \sum_{k=1}^{c} P_{\varphi}\left(N_{0} f_{0}^{k}+N_{\phi} f^{k}\right)
$$

and $\mathcal{N}$, by construction, is a column conservation matrix (Hangos et al., 1999), so that $1^{T} \mathcal{N}=0$.

For the reactor-separator process we are considering, there are two dissipative subnetworks: one in the mass layer (formed by nodes 3 and 4) and one in the energy layer (formed by nodes 1 and 2). The operators $P_{\varphi}$ and $P_{\psi}$ needed to construct the corresponding inventory network described by Eqns. (9) and (10) are of the form:

$$
P_{\varphi}=\left[\begin{array}{ccc}
1 & 0 & 0 \\
0 & 1 & 1
\end{array}\right] ; \quad P_{\psi}=\left[\begin{array}{cccc}
1 & 1 & 0 & 0 \\
0 & 0 & 1 & 0 \\
0 & 0 & 0 & 1
\end{array}\right]
$$

These operators are obtained by solving their defining equations $\left(P_{\varphi} N_{\varphi}=0\right.$, $\left.P_{\psi} N_{\psi}=0\right)$. Now, applying $P_{\varphi}$ to Eqn. (5), we have:

$$
\left[\begin{array}{c}
\dot{n}_{\mathcal{I}_{1}} \\
\left(\dot{n}_{3}+\dot{n}_{4}\right)_{\mathcal{I}_{2}}
\end{array}\right]=\left[\begin{array}{ccc}
-1 & 0 & \alpha_{41} \\
1 & -1 & -1
\end{array}\right]\left[\begin{array}{c}
F_{1} \\
F_{3} \\
F_{4}
\end{array}\right]+\left[\begin{array}{l}
1 \\
0
\end{array}\right] F_{0}+\underbrace{\left[\begin{array}{cc}
-1 & 1 \\
0 & 0
\end{array}\right]\left[\begin{array}{l}
W_{1} \\
W_{2}
\end{array}\right]}_{\mathcal{R}}
$$

Using expressions (7) and (8), where:

$n_{\mathcal{I}_{0}}=n_{0}, \quad n_{\mathcal{I}_{1}}=n_{1}, \quad n_{\mathcal{I}_{2}}=n_{3}+n_{4} ; \quad F_{\mathcal{I}_{0}}=F_{0}, \quad F_{\mathcal{I}_{1}}=F_{1}, \quad F_{\mathcal{I}_{2}}=F_{3}+F_{4}$

Eqn. (12) can be re-written in the form:

$$
\left[\begin{array}{l}
\dot{n}_{\mathcal{I}_{0}} \\
\dot{n}_{\mathcal{I}_{1}} \\
\dot{n}_{\mathcal{I}_{2}}
\end{array}\right]=\left[\begin{array}{ccc}
-1 & 0 & \alpha_{20} \\
1 & -1 & \alpha_{21} \\
0 & 1 & -1
\end{array}\right]\left[\begin{array}{l}
F_{\mathcal{I}_{0}} \\
F_{\mathcal{I}_{1}} \\
F_{\mathcal{I}_{2}}
\end{array}\right]+\mathcal{R}
$$

By using operator $P_{\psi}$, the same logic applies to the construction of the corresponding energy inventory network dynamics (Eqn. 10) from Eqn. (6), which for our example becomes:

$$
\left[\begin{array}{c}
\dot{u}_{\mathcal{I} 0} \\
\dot{u}_{\mathcal{I} 1} \\
\dot{u}_{\mathcal{I} 2}
\end{array}\right]=\left[\begin{array}{ccc}
-1 & 0 & \alpha_{20} \\
1 & -1 & \alpha_{21} \\
0 & 1 & -1
\end{array}\right]\left[\begin{array}{l}
p_{\mathcal{I} 0} \\
p_{\mathcal{I} 1} \\
p_{\mathcal{I} 2}
\end{array}\right]+Q
$$




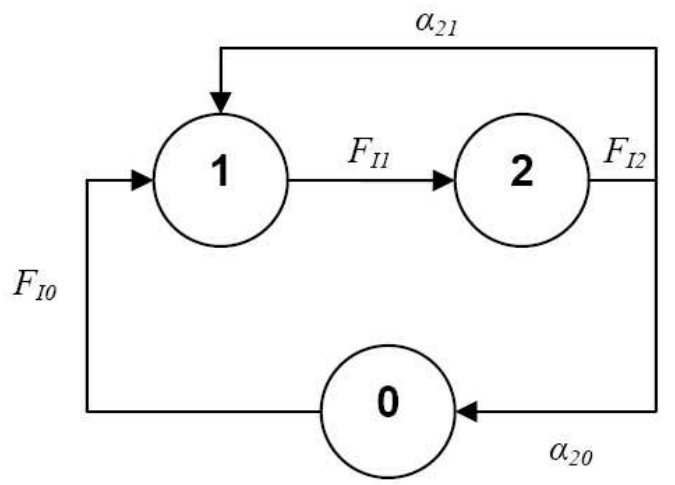

Fig. 3. Mole inventory network representation. Node 0 represents the environment while nodes 1 and 2 the reactor unit and the flash dissipative sub-network, respectively.

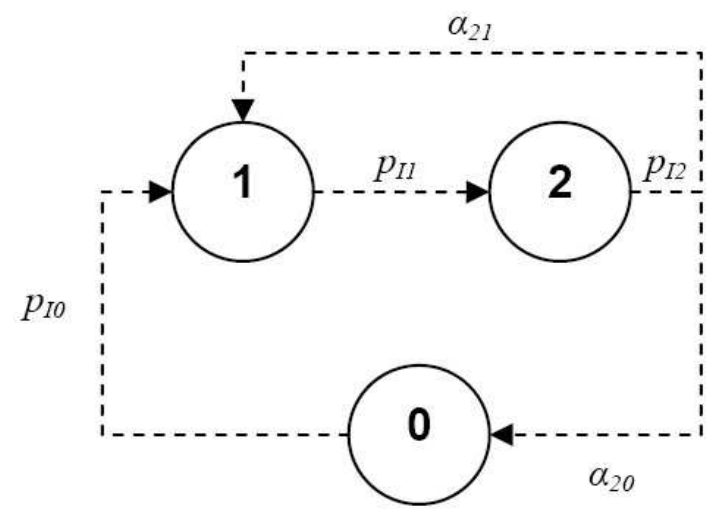

Fig. 4. Energy inventory network representation. Node 0 represents the environment while nodes 1 and 2 the reactor dissipative sub-network and the flash unit, respectively.

A graphical representation of the resulting mass and energy inventory networks is presented in Figure 3 and Figure 4, respectively.

Alternative network representations can be derived from the mole inventory network dynamics (9)-(10). In this way, the corresponding mass inventory network representation for a dissipative subnetwork $\mathcal{D}$ can be easily obtained by defining the following transformations:

$$
\begin{aligned}
m_{\mathcal{D}} & =\sum_{i \in \mathcal{D}} \sum_{k=1}^{c} \sigma^{k} n_{\mathcal{D}}^{k} \equiv \sum_{i \in \mathcal{D}} m_{i} \\
\phi_{\mathcal{D}} & =\sum_{i \in \mathcal{D}} \sum_{k=1}^{c} \sigma^{k} f^{k} \equiv \sum_{i \in \mathcal{D}} \phi_{i}
\end{aligned}
$$

where $\sigma^{k}$ denotes the molecular weight of component $k$, while $m_{i}$ and $\phi_{i}$ rep- 
resent the hold-up and flow, in units of mass, associated to each node in the dissipative sub-network. Using relations (15) and (16), Eqn (9) becomes:

$$
\dot{m}_{\mathcal{I}}=\mathcal{N} \phi_{\mathcal{I}} \quad m_{\mathcal{I}}, \phi_{\mathcal{I}} \in \mathbb{R}^{+\ell}
$$

with $m_{\mathcal{I}}, \phi_{\mathcal{I}} \in \mathbb{R}^{+\ell}$. Note that the reaction term $\mathcal{R}$ in (17) disappears when projecting mole inventories onto mass inventories since the latter is a conserved property.

\section{$3 \quad$ Hierarchical Design of Decentralized Controllers}

In this Section, we present the basic ingredients of conceptual inventory and intensive variable control (the next steps in the systematic control design procedure), which exploit the underlying structure of the process networks previously discussed, and combines them with a number of results from irreversible thermodynamics. Essentially, thermodynamics gives us a function -the entropy of the system- which has a definite curvature (concavity) over compact regions of the state space constrained by constant total mass and energy. Moreover, the function has a well-defined maximum in those regions. Such function will be the one employed to derive natural storage and Lyapunov function candidates of use in designing controllers for stabilizing the intensive variables of the network (i.e. temperature, pressure and concentration).

\subsection{Thermodynamic foundations of process networks}

To start with, let us consider that each node in the process network with volume $\nu_{j}$ is equipped with a continuous and twice differentiable scalar function $S_{j}\left(z_{j}, \nu_{j}\right): \mathbb{R}^{+(c+2)} \mapsto \mathbb{R}$ with the following properties:

(1) $S_{j}\left(z_{j}, \nu_{j}\right)$ is a first order homogeneous function in all their arguments so that

$$
S_{j}=w_{j}^{T} z_{j}+w_{\nu} \nu_{j}
$$

being $w^{T}$ the vector of intensive variables

$$
w=\frac{\partial S}{d z}=\left[\frac{1}{T},-\frac{\mu^{1}}{T}, \ldots,-\frac{\mu^{c}}{T}\right]
$$

and $w_{\nu}=\frac{P}{T}$ the intensive counterpart of the node volume $\nu_{j}$.

(2) $S_{j}\left(z_{j}, \nu_{j}^{*}\right)$ is strictly concave with respect to the vector $z_{j}$.

Note that the function $S_{j}$, as defined in (18), coincides with the classical thermodynamic entropy (Callen, 1985). From Property 1, it follows that entropy 
is an additive function so that the total entropy of a process network is of the form $S=\sum_{j=1}^{\ell} S_{j}\left(z_{j}, \nu_{j}\right)$, where summation extends over the set of dissipative sub-networks.

Property 2 ensures that for a given constant pair $(m, u, \nu)$ and for every $j \in \mathcal{D}$, the function $S$ has a maximum over the convex set:

$$
\Lambda(\mathcal{D})=\left\{\left(z_{j}, \nu_{j}\right) \in \mathbb{R}^{+(c+2)} \mid \quad \sum_{j \in \mathcal{D}} \nu_{j}=\nu, \sum_{j \in \mathcal{D}} u_{j}=u, \sum_{j \in \mathcal{D}} \sum_{k=1}^{c} \sigma^{k} n_{j}^{k}=m\right\}
$$

The entropy balance is obtained by computing the time derivative of $S$ along (3)-(4) so that:

$$
\frac{d S}{d t}=\frac{\partial S}{\partial z} \frac{d z}{d t}=w^{T} \frac{d z}{d t}=P_{s}+\Phi_{s}\left(\mu_{j}, \phi\right)
$$

$P_{s}$ corresponds with the entropy produced by the network, being of the form:

$$
P_{s}=\sum_{i} \sum_{j}\left[\varphi_{i j} \psi_{i j}\right]\left(w_{i}-w_{j}\right) \geq 0
$$

which according to the second law is a non-negative quantity. In addition, $\Phi_{s}\left(\mu_{j}, \phi\right)$ in $(21)$ denotes the entropy flow through the network.

For closed systems $\left(\phi_{\mathcal{I}}=0\right)$, the material and energy inventories are constant, as it can be easily shown by noting that:

$$
\phi_{\mathcal{I}}=0 \Rightarrow F_{\mathcal{I}}=\sum_{i \in \mathcal{D}} \sum_{k} f_{\mathcal{I}}^{k}=0 \quad \text { for } \quad k=1, \ldots, c
$$

and $p(0)=0$, so that Eqns. (10) and (17) become $\dot{m}_{\mathcal{I}}=\dot{u}_{\mathcal{I}}=0$. Consequently, the states of the system remain in the convex set $\Lambda$ defined by $(20)$, with $(m, u$, $\nu)$ being the initial values for the total mass, energy and volume, respectively. Since for this case $\Phi_{s}\left(\mu_{j}, 0\right)=0$ and by the second law $P_{s}$ never becomes negative, $S$ is maximized in $\Lambda$. The maximum is attained at the equilibrium state $\left(z_{j}^{\prime}\right)$ of the network, defined by:

$$
\left(z_{j}^{\prime}\right)_{j=1}^{n}=\operatorname{Arg} \underset{\Lambda}{\operatorname{Max} S}
$$

which also corresponds with that of minimum entropy production i.e. $P_{s}\left(z_{j}^{\prime}\right)=$ 0 .

In general, boundedness of the entropy cannot be guaranteed in open networks since the flows exchanging mass and energy with the environment might produce fluctuations of the inventories thus destroying $\Lambda$. Nevertheless, regions 
$\Lambda$ as defined in (20) can still be meaningful in open networks, provided that material and energy fluxes through the system are able to force their corresponding inventories to be kept at some given constant reference.

Whenever this is the case, process networks can be easily connected with passivity as defined in systems theory (see, for instance, Van der Shaft, 2000). A dynamic system is said to be passive if it is equipped with a real function $V$ bounded from below and related to the inputs and outputs of the system $(u, y)$ by the following inequality:

$$
V(t+\tau) \leq V(t)+\int_{t}^{t+\tau} \phi(u, y) d t
$$

for any $\tau>0$. Function $V$-known as the storage- is usually related with a norm of the states and reflects the total amount of "energy" stored in the system. The term $\phi(u, y)$, known as the supply, represents the net amount of energy exchanged between the system and the environment. The particular structure of inequality (24) allows the construction of control laws $u(y)$ which by making the supply term negative definite, will constrain the amount of energy stored. This, in turn, will ensure the states to remain bounded, or even to asymptotically converge to arbitrary references.

The connections between passivity and process networks can now be easily established by comparing Eqn. (24) with the time integrated version of Eqn. (21) where the storage function now reads $V=S_{\max }-S>0$, being $S_{\text {max }}$ the maximum attained in $\Lambda$, and $S=\sum_{j \in D} S_{j}$.

Moreover, once in the convex set, the existence of a bijective map between the vector of intensive properties $w_{j}$ and the vector of densities $\rho_{j}=\nu_{j}^{-1} z_{j}$ is well established. In fact, by making use of the Newton's theorem for vectorial fields we have that:

$$
w(z)-w\left(z^{*}\right)=Q\left(z-z^{*}\right)
$$

with

$$
Q=\int_{0}^{1} M\left(z^{*}-\varepsilon\left(z-z^{*}\right)\right) d \epsilon
$$

where $z^{*} \in \Lambda$ is an arbitrary reference vector, $\varepsilon \in[0,1]$ is a scalar parameter and the integration is carried out element-wise. Matrix $M$-which corresponds with the Hessian of the entropy- is of the form:

$$
M_{i j}=\frac{\partial w_{i}}{\partial z_{j}}=\frac{\partial^{2} S}{\partial z_{i} \partial z_{j}}<0
$$

Since $M$ is negative definite, it follows that $Q$ is negative definite and thus the relationship between $w-w^{*}$ and $z-z^{*}$ in (25) is one-to-one and onto for arbitrary $z^{*}$. 
Such mapping between intensive variables and densities has been employed by Alonso and Ydstie (2001) to derive stability conditions for distributed process systems. Hangos et al. (1999) made use of the same bijective map to derive structural stability conditions for process plants under constant holdup conditions. However, it must be highlighted that in order to ensure such mapping, a region of constant mass and energy inventories of the form (20) needs be constructed first by appropriate inventory control loops. This point is discussed next.

\subsection{Conceptual inventory control design}

As discussed previously, concavity can only be attained once the network states are in the set $\Lambda$ defined in Eqn. (20). This fact motivates a hierarchical control design decomposition in which mass and energy inventory controllers are first designed to ensure that the network states will remain in $\Lambda$. This is what we will refer from now on as conceptual inventory control. In particular, these mass and energy inventory control layers consist of linear proportional controllers of the form:

$$
\begin{gathered}
\phi_{\mathcal{I}}=\phi_{\mathcal{I}}^{*}+\omega_{m}\left(m_{\mathcal{I}}-m_{\mathcal{I}}^{*}\right) \\
p_{\mathcal{I}}=p_{\mathcal{I}}^{*}+\omega_{u}\left(u_{\mathcal{I}}-u_{\mathcal{I}}^{*}\right)
\end{gathered}
$$

for some $\phi_{\mathcal{I}}^{*}$ being in the null space of $\mathcal{N}$, i.e. $\mathcal{N} \phi_{\mathcal{I}}^{*}=0 . \omega_{n}$ and $\omega_{u}$ in (28) and (29) are appropriate gain matrices constructed in such a way that the real part of the eigenvalues associated to $\mathcal{N} \omega_{m}$ and $\mathcal{N} \omega_{u}$ are negative.

By defining the deviation variables $\bar{m}_{\mathcal{I}}=m_{\mathcal{I}}-m_{\mathcal{I}}^{*}, \bar{u}_{\mathcal{I}}=u_{\mathcal{I}}-u_{\mathcal{I}}^{*}$ and applying (28)-(29) to the mass and energy inventory networks (10) and (17), we get:

$$
\begin{aligned}
& \dot{\bar{m}}_{\mathcal{I}}=\mathcal{N} \omega_{m} \bar{n}_{\mathcal{I}} \\
& \dot{\bar{u}}_{\mathcal{I}}=\mathcal{N} \omega_{u} \bar{u}_{\mathcal{I}}
\end{aligned}
$$

Integration of these expressions over time then results in:

$$
\begin{aligned}
\bar{m}_{\mathcal{I}} & =\bar{m}_{\mathcal{I}}(0) e^{\mathcal{N} \omega_{m} t} \\
\bar{u}_{\mathcal{I}} & =\bar{u}_{\mathcal{I}}(0) e^{\mathcal{N} \omega_{u} t}
\end{aligned}
$$

¿From these expressions, it follows that $m_{\mathcal{I}} \rightarrow m_{\mathcal{I}}^{*}$ and $u_{\mathcal{I}} \rightarrow u_{\mathcal{I}}^{*}$ exponentially fast. Integral action could be also added in Eqns. (28)-(29) to improve performance without substantially altering the stability properties of the closed loop network (Farschman et al., 1998). At this point, it should be mentioned that although control is usually exerted on a mass (or volumetric) basis, the design of a stabilizing control scheme for total mole inventory (for instance 
on a gas-phase sub-network) would follow a similar line of arguments in the corresponding mole inventory representation.

In some situations, mass inventory control is enough to guarantee the stability of both mass and energy inventories around a given reference. In order to show this, we first introduce the following result on the stability properties of a particular class of dynamic system:

Lemma 3.1 Let us have a dynamic system:

$$
\dot{x}=\mathcal{A} D(y) x \quad x \in \mathbb{R}^{n}
$$

where $\mathcal{A}$ is a row and column (Kirchoff) conservation matrix (Hangos et al., 1999), $y \in R^{+n}$ is a constant vector and $D(y)=\operatorname{Diag}(y)$.

Then, the origin $(x=0)$ is a stable equilibrium point for the system (34).

Proof: The proof follows by defining a Lyapunov function candidate:

$$
W=\frac{1}{2} x^{T} D(y) x
$$

and computing its time derivative along (34), then:

$$
\dot{W}=x^{T} D(y) \mathcal{A} D(y) x
$$

Since $\mathcal{A}$ is a Kirchoff conservation matrix, it is negative semidefinite (Hangos et al., 1999). Thus, $\dot{W} \leq 0$ and the result follows by applying standard Lyapunov methods (Slotine and Li, 1991).

Proposition 3.2 For a given dissipative sub-network $\mathcal{D}$, the following proportional controllers:

$$
\phi_{i}=\phi_{i}^{*}+\omega_{i}\left(m_{i}-m_{i}^{*}\right)
$$

with reference flows and inventories satisfying:

$$
\frac{\phi_{i}^{*}}{m_{i}^{*}}=\frac{\phi_{\mathcal{D}}^{*}}{m_{\mathcal{D}}^{*}}
$$

for every node $i \in \mathcal{D}$, will stabilize mass and energy inventories around a given reference $\left(m_{\mathcal{I}}^{*}, u_{\mathcal{I}}^{*}\right)$.

Proof: Controllers of the form (37) will stabilize the mass inventory since the summation of the mass flows for every $i \in \mathcal{D}$ and for every sub-network would lead to inventory control laws of the form (28) providing exponential convergence of the mass inventories -see (32). Since $m_{\mathcal{I}} \rightarrow m_{\mathcal{I}}^{*}$, we also have that $\phi_{\mathcal{I}} \rightarrow \phi_{\mathcal{I}}^{*}$. 
In order to show convergence of the energy inventory, let us note that the energy flow associated to each node $i \in \mathcal{D}$, and thus to each sub-network, can be expressed as:

$$
p_{i}=\frac{u_{i}}{m_{i}^{*}} \phi_{i}^{*} \quad p_{\mathcal{D}}=\sum_{i \in \mathcal{D}} \frac{u_{i}}{m_{i}^{*}} \phi_{i}^{*}
$$

Combining (38) with (39), allows us to re-write the total energy inventory flow as:

$$
p_{\mathcal{I}}=D\left(\phi_{\mathcal{I}}^{*}\right) D\left(m_{\mathcal{I}}^{*}\right)^{-1} u_{\mathcal{I}}
$$

Substituting (40) into (10) and defining the inventory in deviation form as $\bar{u}_{\mathcal{I}}=u_{\mathcal{I}}-u_{\mathcal{I}}^{*}$, for a given reference, $u_{\mathcal{I}}^{*}$ we get:

$$
\dot{\bar{u}}_{\mathcal{I}}=\mathcal{N} D\left(\phi_{\mathcal{I}}^{*}\right) D\left(m_{\mathcal{I}}^{*}\right)^{-1} \bar{u}_{\mathcal{I}}
$$

Since, as discussed in Section $2, \mathcal{N}$ is a column conservation matrix and $\mathcal{N} \phi_{\mathcal{I}}^{*}=0, \mathcal{N} D\left(\phi_{\mathcal{I}}^{*}\right)$ is a row and column (Kirchoff) conservation matrix. The result then follows by making use of Lemma 3.1 with $\mathcal{A} \equiv \mathcal{N} D\left(\phi_{\mathcal{I}}^{*}\right)$ and $D(y) \equiv$ $D\left(m_{\mathcal{I}}^{*}\right)^{-1}$.

\subsection{Realization of the conceptual inventory control structure}

The formalism concerning the conceptual inventory control design presented must be translated into suitable control loops, constituting the next step in the systematic control design procedure. For this purpose, the available inputs and outputs of the system are used since, as shown previously in this work, the total inventory fluxes can be the result of combining multiple convective outflow streams. As a consequence, the inventory control law has to be obtained as a combination of control loops implemented over the real manipulated variables available in the process. This is what we will refer from now on as control loop realization.

Additional inventory control loops for mass and energy in every node belonging to the dissipative subnetwork $\mathcal{D}$ are needed. The reason is that the control laws, as defined in Eqns. (28)-(29), do not necessarily ensure the convergence of the node states $\left(z_{j} ; \forall j \in \mathcal{D}\right)$ to the interior of the convex set $\Lambda$ since the node states could in some instances evolve to the bounds where $z_{j}=0$. In order to avoid this, we propose control laws for the inventories in each node analogous to (28)-(29), and of the form:

$$
\begin{aligned}
& \phi_{L}=\phi_{L}^{*}+\omega_{L}\left(m_{L}-m_{L}^{*}\right) \\
& \phi_{V}=\phi_{V}^{*}+\omega_{L}\left(m_{V}-m_{V}^{*}\right)
\end{aligned}
$$

Note that these last expressions are for the case of a liquid-vapor dissipative subnetwork as the one formed by nodes 3 and 4 in Figure 2 . It follows that the summation of Eqns. (42) and (43) results in $\phi_{\mathcal{I}}=\phi_{\mathcal{I}}^{*}+\omega_{m}\left(m_{\mathcal{I}}-m_{\mathcal{I}}^{*}\right)$ for 
$\omega_{L}=\omega_{V}$, ensuring the convergence of both total and node mass inventories. An analogous result can be obtained for the case of the energy inventory control layer.

\subsection{The intensive variable control issue}

Despite convergence of the system states to the interior of the convex set is ensured by using inventory control, it is known that complex behavior such as multiplicities or oscillatory phenomena can appear in $\Lambda$, in terms of the intensive variables.

Based on the properties of the entropy function in compact regions, Alonso and Ydstie (2001) proposed a formal framework for stability analysis and stabilizing control design of dissipative process systems. In this way, the framework allows the construction of Lyapunov function candidates which can be used to design new nonlinear robust controllers or to test the stabilizing capabilities of existing control schemes. A typical candidate is that defined by the difference between entropy and the supporting hyperplane at the reference, which takes the form:

$$
b\left(z, z^{*}\right)=\left[S\left(z^{*}\right)+w\left(z^{*}\right)^{T}\left(z-z^{*}\right)\right]-S(z) \geq 0
$$

where $w^{T}$ is the vector of intensive variables as defined by (19). Recently, and based on the same concept of availability, Jillson and Ydstie (2005) and Antelo et al. (2005) have developed a more general storage candidate $R\left(z, z^{*}\right)$ based on the concept of available work $(A)$ defined by Keenan (1951). This function is of the form:

$$
R\left(z, z^{*}\right)=A(z)+A\left(z^{*}\right)=-\left(z-z^{*}\right)^{T}\left(w-w^{*}\right) \geq 0
$$

where the pairs $(z, w)$ and $\left(z^{*}, w^{*}\right)$ are defined by the vector of states and of intensive variables for the system and the reference, respectively, and $A(z)=$ $-\left(w-w^{*}\right)^{T} z$ coincides with the availability. Note that $R\left(z, z^{*}\right)$ is positive definite, since as shown in Section $3.1\left(z-z^{*}\right)$ and $\left(w-w^{*}\right)$ are related through a negative definite matrix $Q$ (see Eqn. 26).

Thus, by designing intensive variable control schemes making the time derivative of (44) or (45) negative, Lyapunov stability can be concluded for the intensive variables. Similar arguments were employed by Farschman et al. (1998) and Hangos et al. (1999) to ensure convergence of process states to their desired set points. As we will discuss next, this framework -forcing the negativity condition over the entropy balance (21) by modifying the entropy flux- will be the one adopted to design stabilizing temperature and concentration controllers for the reactor network. 


\section{Control of Chemical Reactors}

The proposed hierarchical control design methodology is now applied to the case of an isolated jacketed non-isothermal CSTR, similar to the one considered in the process presented in Figure 1. This exothermic reactor was widely studied in the literature as an example of nonlinear system exhibiting multiplicities (Kevrekidis et al., 1986; Cordonier et al., 1990), and as a good benchmark problem to test stabilizing control structures (see, for instance, Alvarez et al., 1991; Alonso and Banga, 1998). Note that, in our case, and in order to make the system compatible with physics, the exothermic reaction $A$ $\leftrightarrow B$ is assumed to be reversible, although highly displaced to the right. The reaction rate $\rho$ is defined as:

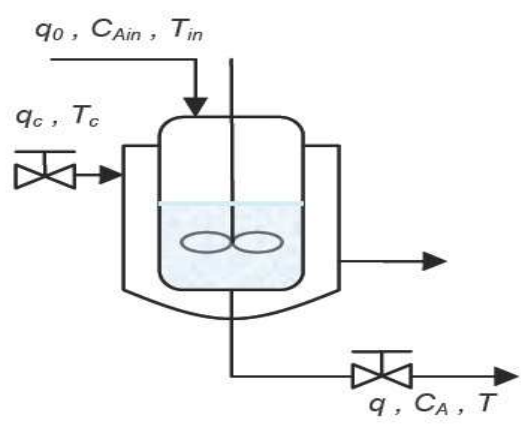

Fig. 5. The jacketed CSTR reactor scheme

$$
\rho=k_{1} C_{A}-k_{2} C_{B}=C_{A} e^{\left(a_{1}-\frac{b_{1}}{T}\right)}-C_{B} e^{\left(a_{2}-\frac{b_{2}}{T}\right)}
$$

where $k_{1}$ and $k_{2}$ correspond with the direct and inverse kinetic constants, respectively, obeying an Arrhenius-type temperature dependance. For the present example, it was assumed that $a_{1}=a_{2}$, and $b_{2}=3 b_{1}$. Under these assumptions, Eqn. (46) can be approximated by an quasi-irreversible first order reaction kinetic expression:

$$
\rho \approx C_{A} e^{\left(a_{1}-\frac{b_{1}}{T}\right)}
$$

As a starting point in the control design procedure, we apply the process networks formalism developed in Section 2 to represent the reactor and define its constitutive mass and energy inventory layers. Figure 6 shows the corresponding associated network to the reactor. It consists of two nodes connected by a dissipative heat transfer term plus extra mass and energy convective flows connecting the nodes with the environment. The inventory networks derived from this fundamental network are presented in Figure 7. The dynamic mass and energy balances are of the form:

\section{(1) Node 1:}




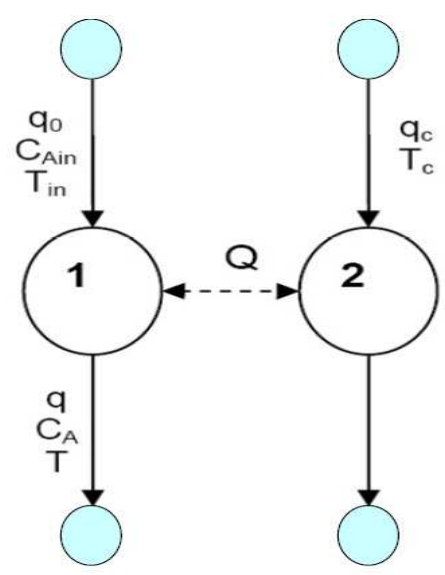

Fig. 6. Reactor process network. Nodes 1 and 2 represent the reactor and the jacket, respectively. Solid nodes represent the environment

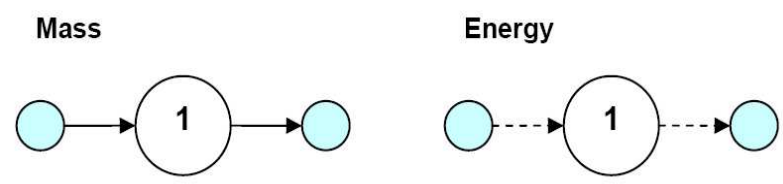

Fig. 7. Reactor mass and energy inventory networks

- Balance of component $A$ :

$$
\frac{d n_{1}^{A}}{d t}=q_{0} C_{A}^{i n}-q C_{A}-\rho \nu
$$

- Balance of component $B$ :

$$
\frac{d n_{1}^{B}}{d t}=-q C_{B}+\rho \nu
$$

\section{- Energy Balance:}

$$
\frac{d U_{1}}{d t}=h_{A}\left(T_{i n}\right) C_{A}^{i n} q_{0}-\left(h_{A}(T) C_{A}+h_{B}(T) C_{B}\right) q-Q\left(T, T_{c}\right)
$$

The nomenclature used in these balances is explained in Table 4.1.

(2) Node 2: In this node, since neither component $A$ nor $B$ are present, only the energy balance is considered:

\section{- Energy Balance:}

$$
\frac{d U_{2}}{d t}=Q\left(T, T_{c}\right)
$$

with

$$
Q=U_{c} A\left(T-T_{c}\right)
$$


Table 4.1. Parameters and variables of the reactor model

\begin{tabular}{|c|c|c|}
\hline Parameters/Variables & Definition & Units \\
\hline$q_{0}$ & Reactor inflow & $\frac{l}{s}$ \\
\hline$q$ & Reactor outflow & $\frac{l}{s}$ \\
\hline$\rho$ & Reaction rate & $\frac{m o l}{l \cdot s}$ \\
\hline$\nu$ & Reactor volume & $l$ \\
\hline$C_{A}$ & Concentration of $\mathrm{A}$ in the reactor & $\frac{m o l}{l}$ \\
\hline$C_{A_{\text {in }}}$ & Concentration of $\mathrm{A}$ in the reactor inlet & $\frac{m o l}{l}$ \\
\hline$h_{k}\left(T_{j}\right)$ & Enthalpy of component $k$ at temperature $T_{j}$ & $\frac{J}{m o l}$ \\
\hline$T$ & Reactor temperature & $K$ \\
\hline$T_{i n}$ & Reactor inlet temperature & $K$ \\
\hline$T_{c}$ & Coolant temperature & $K$ \\
\hline$Q$ & Heat exchanged in the reactor & $\frac{J}{s}$ \\
\hline$U_{c}$ & Heat transfer coefficient & $\frac{J}{m^{2} \cdot K \cdot s}$ \\
\hline$A$ & Heat transfer area & $m^{2}$ \\
\hline$\Delta h^{*}$ & Reaction heat & $\frac{J}{m o l}$ \\
\hline$d$ & Liquid density & $\frac{m o l}{l}$ \\
\hline$c_{p}$ & Liquid heat capacity & $\frac{J}{\mathrm{~mol} \cdot \mathrm{K}}$ \\
\hline$R$ & Gas constant & $\frac{J}{\mathrm{~mol} \cdot \mathrm{K}}$ \\
\hline
\end{tabular}

Note that the algebraic structure underlying these fundamental dynamics of the network is equivalent to Eqns. (3) and (4) by considering that:

$f_{0}^{k}=q_{0} \cdot C_{k_{i n}} ; \quad f^{k}=q \cdot C_{k} ; \quad p_{0}=\sum_{k=1}^{c} h_{k}\left(T_{i n}\right) \cdot q_{0} \cdot C_{k_{i n}} ; \quad p=\sum_{k=1}^{c} h_{k}(T) \cdot q \cdot C_{k}$

with $c=A, B$.

\subsection{Mass and energy inventory control}

The next step in the systematic control design is the application of the conceptual mass and energy inventory control approach (Section 3.2) to the reactor network depicted in Figure 6 so to drive the network states inside region $\Lambda$. For the mass layer case (Figure 7), the inventory loop is closed by considering the total inventory outflow leaving node 1 as the manipulated variable. For the energy case, it can be shown that there only exists an available degree of 
freedom (the inventory inflow to node 1). However, the total energy inventory outflow from node 1 is formed by the summation of two flows present in the dissipative sub-network (Eqn. 8):

- the energy flow associated to the convective mass flow leaving the reactor, used to control the mass layer.

- the energy flow corresponding to the coolant stream.

Since there is not available valve at the reactor inlet, the energy flow is chosen as the manipulated variable candidate to control the energy inventory.

As explained in Section 3.3., the next step consists of the realization of this conceptual design over the available degrees of freedom of the system. The result is shown in Figure 8:

- The mass inventory control results in a level control (LC) loop since the level is directly related with the total mass inventory. This loop sets up the residence time in the reactor by using the reactor outflow $(q)$ as the manipulated variable.

- In order to implement the energy inventory control, an internal energy observer is designed to estimate the energy content in the reactor by using temperature measurements. The reason is that the internal energy cannot be directly measured. Then, this estimate is used in the flow control (FC) loop acting over the coolant flow rate.

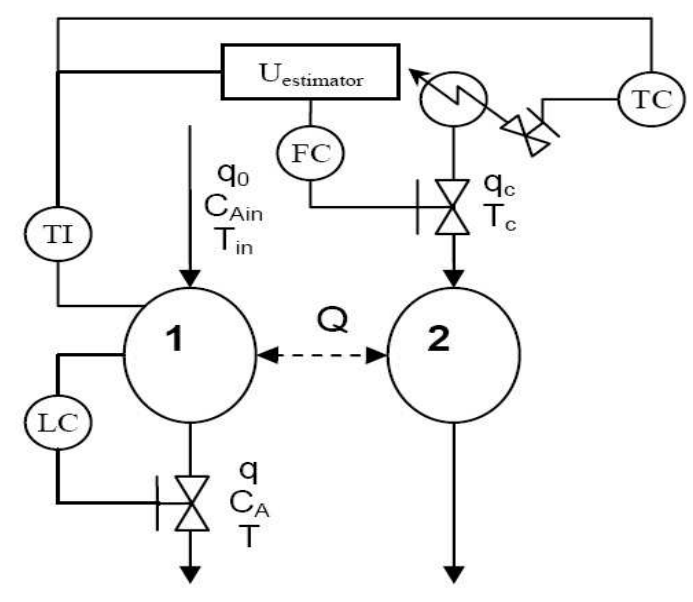

Fig. 8. The proposed control structure (control realization)

As discussed in previous Section, the proposed control structure (Figure 8) guarantees the convergence of the system to the compact set $\Lambda$ (Eqn. 20). For the reactor example, this convex set is defined as the intersection of the plane of constant internal energy, corresponding to the nominal value for this inventory $\left(U=8.36 e^{5} J\right)$, and the straight line satisfying the constant mass condition $m_{A}+m_{B}=18 \mathrm{~kg}$. The resulting convex region is presented in Figure 
9 (dashed line). It must be pointed out that the total mass condition implies that the volume $(\nu)$ will be constant since the density of both components is considered equal to the water density $\left(d_{A}=d_{B}=d=1000 \frac{g}{l}\right)$.

However, as pointed out in Section 3, the proposed inventory control structure does not necessarily ensure the stability of the intensive variables (concentration and temperature) into this convex region. Such behavior can be easily detected by the following thermodynamic analysis.

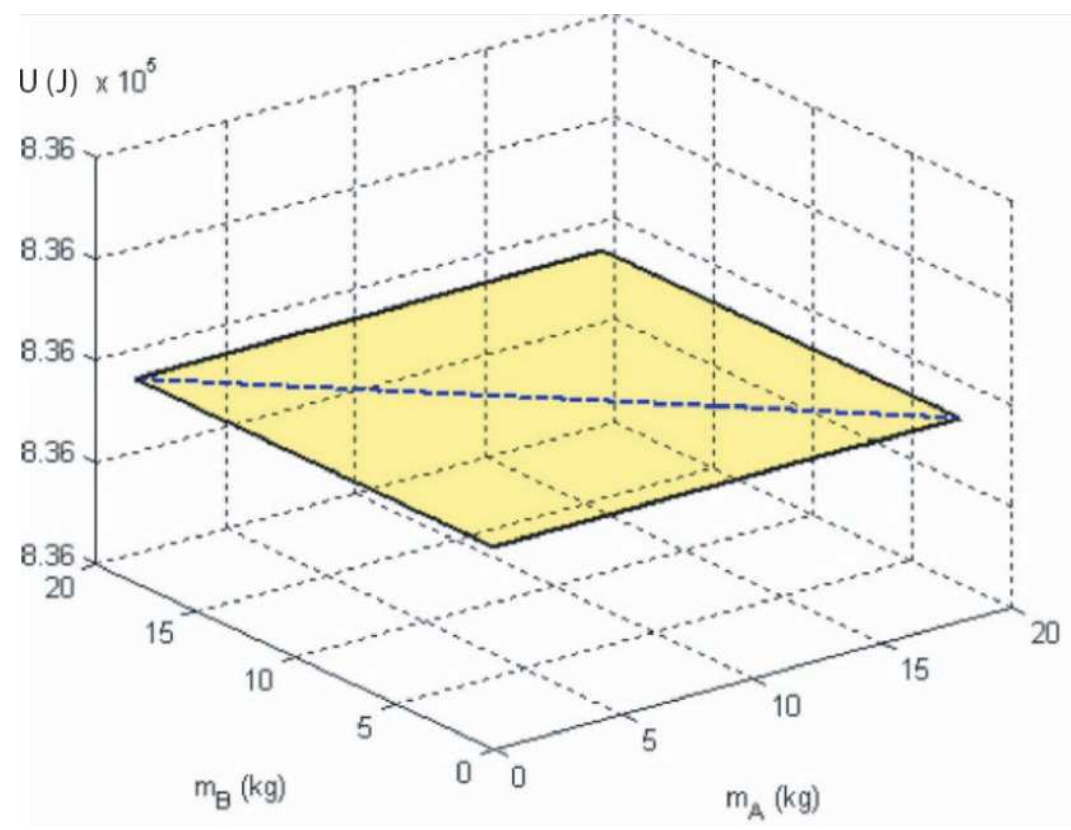

Fig. 9. Convex set $(\Lambda)$ representation for the reactor. $\Lambda$ is the dashed line resulting from the intersection of the constant energy inventory plane $\left(U=8.36 e^{5} \mathrm{~J}\right)$ and the straight line of constant mass condition $\left(m_{A}+m_{B}=18 \mathrm{~kg}\right)$.

\subsection{Thermodynamic analysis of the reactor network}

For the reactor case considered, the multiple steady states that exist into the convex region $(\Lambda)$ can be stated and analyzed by using the entropy balance (Eqn. 21) associated to the network presented in Figure 6. To that purpose, consider the Gibbs-Duhem Equation applied to each node:

$$
\begin{aligned}
& d_{\lambda} S_{1}=\frac{1}{T_{1}} d_{\lambda} U_{1}+\frac{P_{1}}{T_{1}} d_{\lambda} \nu_{1}-\sum_{k} \frac{\mu_{1}^{k}}{T_{1}} d_{\lambda} n_{1}^{k} \\
& d_{\lambda} S_{2}=\frac{1}{T_{2}} d_{\lambda} U_{2}+\frac{P_{2}}{T_{2}} d_{\lambda} \nu_{2}-\sum_{k} \frac{\mu_{2}^{k}}{T_{2}} d_{\lambda} n_{2}^{k}
\end{aligned}
$$

where $\lambda$ is a given variation parameter which, in our case, corresponds with time, and $\mu_{j}^{k}$ is the chemical potential of component $k$ in node $j$ (with $j=$ $\{1,2\})$. For both nodes, we assume that there are not changes in the volume 
$\left(d_{t} \nu=0\right)$ as a consequence of the considered mass inventory control. In addition, it is considered that $d_{t} n_{2}^{k}=0$ in node 2 , since neither chemical reaction nor mass transfer takes place. With these assumptions, Eqn. (53) becomes:

$$
\begin{aligned}
& d_{t} S_{1}=\frac{1}{T_{1}} d_{t} U_{1}-\sum_{k} \frac{\mu_{1}^{k}}{T_{1}} d_{t} n_{1}^{k} \\
& d_{t} S_{2}=\frac{1}{T_{2}} d_{t} U_{2}
\end{aligned}
$$

Due to the energy inventory control implemented, the global internal energy of the network will remain constant, thus $d_{t} U_{1}=-d_{t} U_{2}$. Finally, the entropy variation for the global network is equal to the sum of the entropy variations in the nodes: $d_{t} S_{\text {total }}=d_{t} S_{1}+d_{t} S_{2}$. With these last considerations, Eqn. (54) results in the following general expression for the entropy variation in the reactor:

$$
d_{t} S=\left(\frac{1}{T}-\frac{1}{T_{c}}\right) d_{t} U-\left(\frac{\mu^{A}}{T} d_{t} n^{A}+\frac{\mu^{B}}{T} d_{t} n^{B}\right)
$$

The analytical expression of this balance can be obtained by substituting the balance equations (Eqns. 48-50) into Eqn. (55).

As presented in Eqn. (21), the entropy balance is constituted by two different contributions: entropy production and entropy flow. We define both terms next:

(1) Entropy Production $\left(P_{s}\right)$ : The entropy generation is related to irreversible processes, being its rate a product of dissipative fluxes and thermodynamic driving forces, as shown in Eqn. (22). The analytic expression for the entropy production in the non-isothermal reactor can be defined as follows:

$$
P_{s}=\gamma\left(\frac{1}{T_{c}}-\frac{1}{T}\right)\left(T-T_{c}\right)-\frac{R}{d c_{p}} \rho \frac{\Delta \mu}{T}
$$

with $\Delta \mu=\mu_{B}-\mu_{A}=\log \left(\frac{C_{B}}{C_{A}}\right)-\log \left(\frac{k 1}{k 2}\right)$ and $R$ (the gas constant) equal to $8.314 \mathrm{~J} / \mathrm{mol} \cdot K$. In order to compute these terms, and as presented previously, it is assumed in the model that the fluid has the same physical properties than water, i.e. molecular weight $=18 \mathrm{kmol} / \mathrm{kg}, d=1000 \mathrm{~g} / \mathrm{l}$ and $c_{p}=4.18 \mathrm{~J} / \mathrm{mol} \cdot K$. The right hand side Eqn. (56) consists of two terms:

(a) The first term is due to the heat transfer between nodes. Irreversible heat flux is caused by the difference of temperatures which acts as a thermodynamic driving force.

(b) The second contribution is that related to chemical reaction, being the associated driving force the difference of chemical potentials.

(2) Entropy flux $\left(\Phi_{s}\right)$ : This term is associated with material and energy throughput flows connecting the network with the environment. For the 
reactor case, the entropy flux can be defined as:

$$
\begin{aligned}
\Phi_{s} & =\frac{1}{T}\left[\left[h_{A}\left(T_{i n}\right)-h_{B}(T)\right] C_{A}^{i n}+\left[h_{B}(T)-h_{A}(T)\right] C_{A}\right] q+ \\
& +\frac{\Delta \mu}{T} q\left(C_{A}^{i n}-C_{A}\right)-Q \frac{1}{T_{C}}
\end{aligned}
$$

The enthalpy of the pure components $A$ and $B$ is defined as:

$$
\begin{aligned}
& h_{B}(T)=h_{B}^{*}+c_{p_{B}}^{\prime}\left(T-T^{*}\right) \\
& h_{A}(T)=h_{A}^{*}+c_{p_{A}}^{\prime}\left(T-T^{*}\right) \\
& h_{A}\left(T_{i n}\right)=h_{A}^{*}+c_{p_{A}}^{\prime}\left(T_{i n}-T^{*}\right)
\end{aligned}
$$

In these last expressions, $h_{i}^{*}$ is the enthalpy of component $i$ for a given reference state and $c_{p}^{\prime}$ is the specific heat of the pure components. It is assumed that both components have the same specific heat $\left(c_{p}^{\prime}=c_{p_{A}}^{\prime}=\right.$ $\left.c_{p_{B}}^{\prime}\right)$. From Eqn. (58), it is possible to define:

$$
\begin{aligned}
& h_{B}(T)-h_{A}(T)=\Delta h^{*} \\
& h_{A}\left(T_{i n}\right)-h_{B}(T)=-\Delta h^{*}+c_{p}^{\prime}\left(T_{i n}-T\right)
\end{aligned}
$$

The reaction heat $\left(\Delta h^{*}\right)$ is considered constant. Now, substituting Eqns. (58) and (59) into Eqn. (57), and introducing the dimensionless parameters and the expression for $Q$ previously presented (Eqn. 52), we get the entropy flux for the reactor network:

$$
\begin{aligned}
\Phi_{s} & =\frac{1}{T}\left[\frac{C_{A}^{i n} c_{p_{A}}\left(T_{i n}-T\right)}{d c_{p}}+\beta\left(C_{A}-C_{A}^{i n}\right)\right] \theta- \\
& -\frac{\Delta \mu}{T d c_{p}}\left(C_{A}^{i n}-C_{A}\right) \theta-\frac{\gamma}{T_{c}}\left(T-T_{c}\right)
\end{aligned}
$$

Process entropy and its time derivative (defined by the summation of Eqns. 56 and 60) are depicted in Figure 10 along the manifold of constant mass and energy $\Lambda$ defined by Eqn. (20). In order to represent $d S$ with respect to $C_{A}$, we need the temperature corresponding to each concentration. To that purpose, the stationary energy balance (Eqn. 62) is considered to calculate T. As it can be seen in this Figure, there are three points in $\Lambda$ satisfying the steady-state entropy balance which corresponds to an unstable and two stable stationary points. As presented in Section 3.1, entropy is a concave function that attains its maximum at the equilibrium. For the quasi-irreversible reaction $A \leftrightarrow B$ we are considering, this maximum lies very close to the point $C_{A}=0$. This allows us to plot a proper approximation of $S$ versus $C_{A}$, as shown in Figure 10. On this plot, the stability or instability of the different steady states can be easily established from the curvature of the system entropy and its time 
derivative (Eqn. 21). For instance, the stationary point in Figure (10) which corresponds to $C_{A}=0.0885 \frac{\mathrm{mol}}{\mathrm{l}}$ is stable since any disturbance decreasing the concentration results in a negative sign for $d S$, which makes $S$ to decrease. On the other hand, positive disturbances around this stationary point make $d S$ positive so that $S$ will increase. As a consequence, this point is attainable and stable.

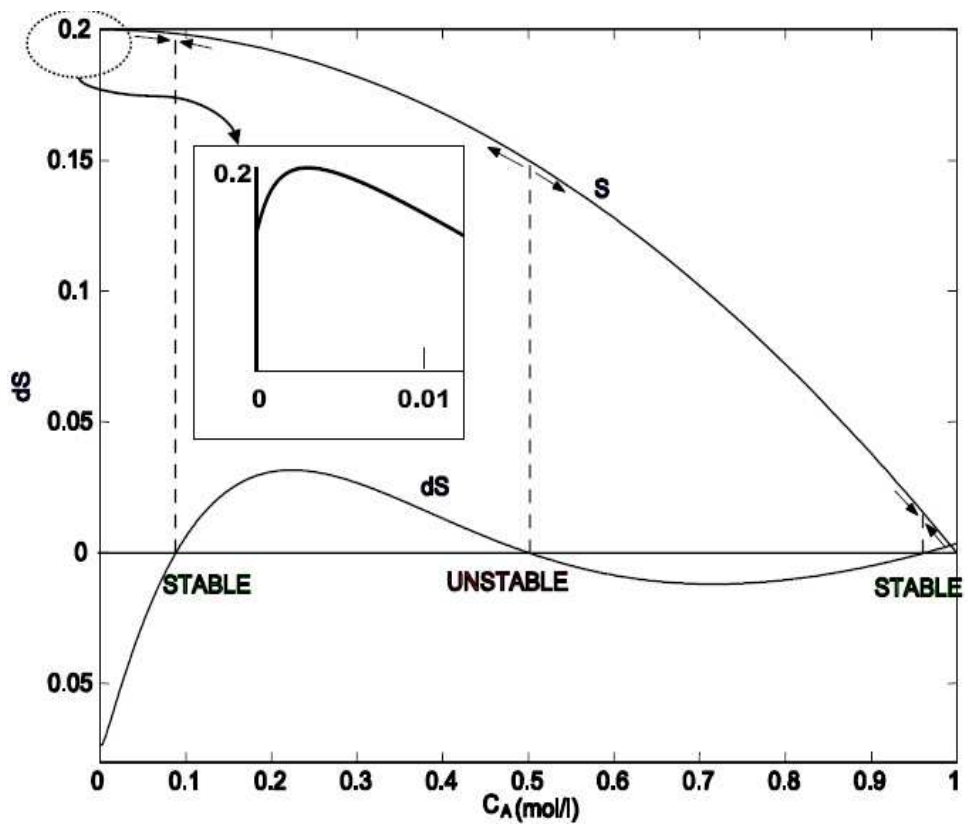

Fig. 10. Steady states stability analysis using $S$. The zoom window shows the maximum of entropy $S$ (very close to $C_{A}=0 \frac{\mathrm{mol}}{\mathrm{l}}$ ). The points where $d S=0$ correspond to the steady states exhibited by the reactor. Stability or instability is determined by the sign of $d S$ and the curvature of $S$.

The multiple steady states inside $\Lambda$, where the system remains on as a result of the proposed inventory control structure, can be also detected by using a classical analysis. This approach is based on the reactor network dynamics (Eqns. 48 - 50) expressed only in terms of intensive variables (concentration and temperature), which for this case take the form:

$$
\begin{gathered}
\dot{C}_{A}=-\rho+\theta\left(C_{A}^{i n}-C_{A}\right) \\
\dot{T}=\beta \rho+\theta\left(T_{i n}-T\right)-\gamma\left(T-T_{c}\right)
\end{gathered}
$$

where $\theta, \beta$, and $\gamma$ are dimensionless physical parameters related with residence time, reaction heat, and heat transfer areas, respectively. They are defined as follows (see Cordonier et al., 1990 or Alonso and Banga, 1998, for further details):

$$
\begin{gathered}
\theta=\frac{q}{\nu} \\
\beta=\frac{-\Delta h}{d c_{p}}
\end{gathered}
$$


Table 4.2. Nominal values for inlet variables and parameters employed for the non-isothermal reactor model

\begin{tabular}{|c|c|c|c|c|c|c|c|c|}
\hline$C_{A}^{\text {in }}(\mathrm{mol} / \mathrm{l})$ & $T_{\text {in }}(K)$ & $a_{1}$ & $a_{2}$ & $b_{1}(K)$ & $b_{2}(K)$ & $\theta(1 / \mathrm{s})$ & $\beta$ & $\gamma$ \\
\hline 1 & 350 & 25 & 25 & 10000 & 30000 & 1 & 200 & 1 \\
\hline
\end{tabular}

$$
\gamma=\frac{U_{c} A}{d \nu c_{p}}
$$

The values considered in the model for the inlet conditions, kinetics and dimensionless parameters, are presented in Table 4.2. Considering these values, the reaction network represented by Eqns. (61) and (62) exhibits the same three steady states detected by applying the thermodynamic analysis, as shown in Figure 11. In this Figure, the trajectories of composition and temperature in the reactor (for different initial conditions) are plotted, showing that different reactor product compositions can be attained depending on the initial conditions. In addition, the geometric place of constant concentration $\left(\dot{C}_{A}=0\right)$ is represented by a dashed curve as a consequence of the existing nonlinear relationship between these intensive variables. It intersects the stationary energy balance $(\dot{T}=0)$ in the three different steady states.

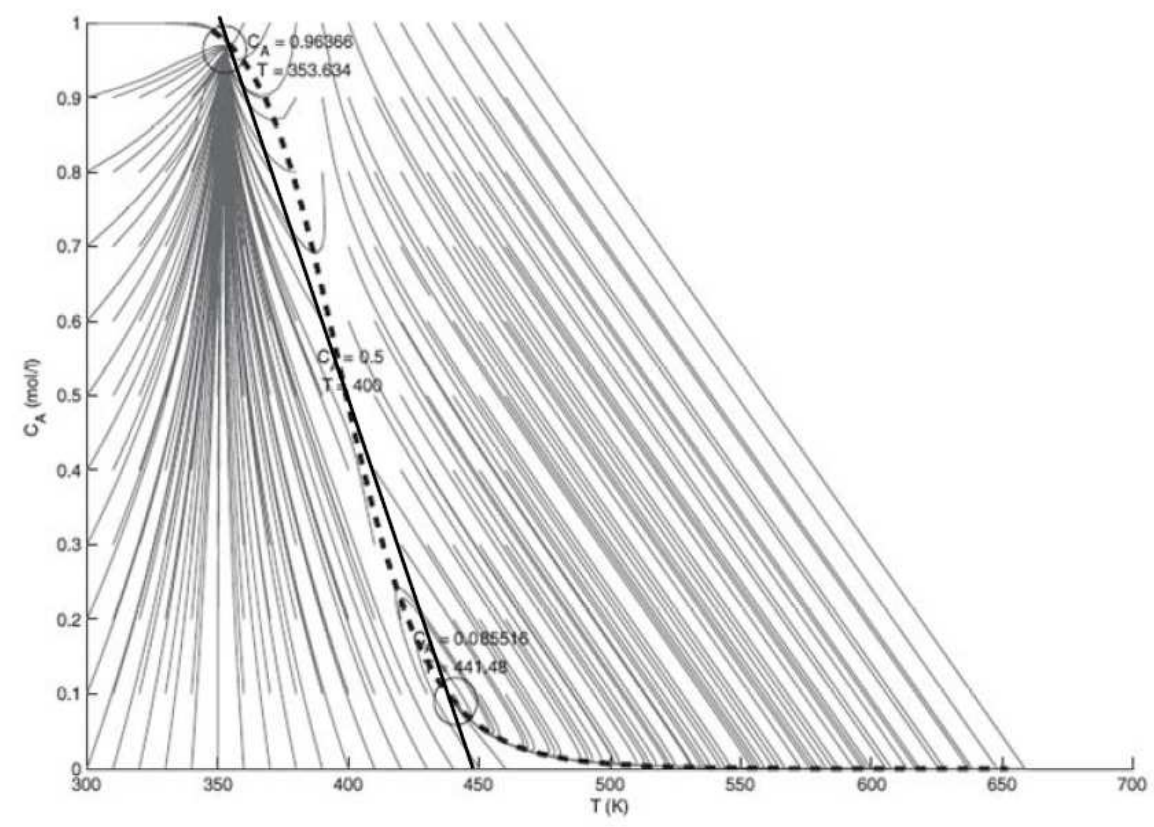

Fig. 11. State Space for the Non-Isothermal CSTR. The dashed and straight lines represent the stationary balances of $C_{A}$ and $T$, respectively. The intersection between these are the three steady states present in the reactor 
In the last step on the control structure design procedure, the inventory control configuration proposed in Section 4.1 is completed with a controller of intensive variable acting on the coolant temperature $T_{c}$. This extra loop, as we will se, ensure the stabilization of the intensive variables (reactor concentration and temperature) to the desired point which, for the reactor example, corresponds to the unstable steady state.

Although different control structures could serve to that purpose, we apply well-known geometric controllers to stabilize the intensive variables: a PI temperature controller and a non-linear exact linearizing control were tested, and state stabilization was demonstrated for both cases. With this intensive variable control considered, the entropy flux (Eqn. 57) is modified as explained in Section 3. As a consequence, a unique steady state in $\Lambda$, where $d S=0$, is obtained.

Let us redefine $C_{A}$ and $T$ as $x_{1}$ and $x_{2}$, respectively, being the model (61)-(62) in input affine form:

$$
\begin{aligned}
& \dot{x}=f(x)+g(x) u \\
& y=h(x)
\end{aligned}
$$

where

$$
\begin{gathered}
f(x)=\left(\begin{array}{c}
-\rho+\theta\left(x_{1}^{i}-x_{1}\right) \\
\beta \rho+\theta\left(x_{2}^{i}-x_{2}\right)-\gamma x_{2}
\end{array}\right) \\
g(x)=\left(\begin{array}{l}
0 \\
\gamma
\end{array}\right) ; \quad h(x)=x_{1}
\end{gathered}
$$

1. PI Control: The selected control law is of the form:

$$
u=x_{2 c}=x_{2 c r}-k\left(x_{2}-x_{2 r}\right)+\frac{1}{\tau_{I}} \int\left(x_{2}-x_{2 r}\right) d t
$$

where $x_{2 c r}$ is the reference temperature for the coolant $(350 \mathrm{~K}), k$ is the controller gain, $T_{2 r}$ is the set point for the reactor temperature and $\tau_{I}$ is the time constant. The controller parameters were adjusted to $k=10$ and $\tau_{I}=58$.

The entropy variation $\left(d S=P_{s}+\phi_{s}\right)$ for this closed loop system is presented in Figure 12. It is clearly demonstrated that the entropy flux term is modified by this intensive variable control, as explained in Section 3.4. This variation can be shown when comparing Figures 13 and 14. As a result, the multiplicities detected in Figure 10 disappear, existing a unique steady state which corresponds to the selected point. Note that this control can also modify the entropy production (Eqn. 56). However, these variations (Figure 15) are quite 
similar for both open and closed loop cases and not significative when compared to changes in the entropy flux (Figure 14).

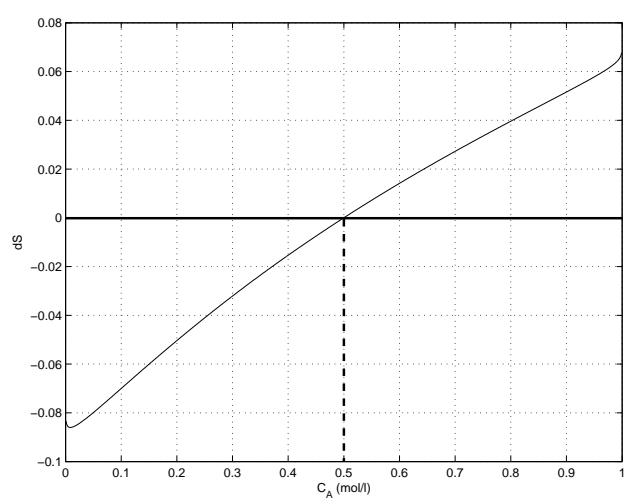

Fig. 12. Entropy variation $(d S)$ for the reactor with a PI control loop for $T$

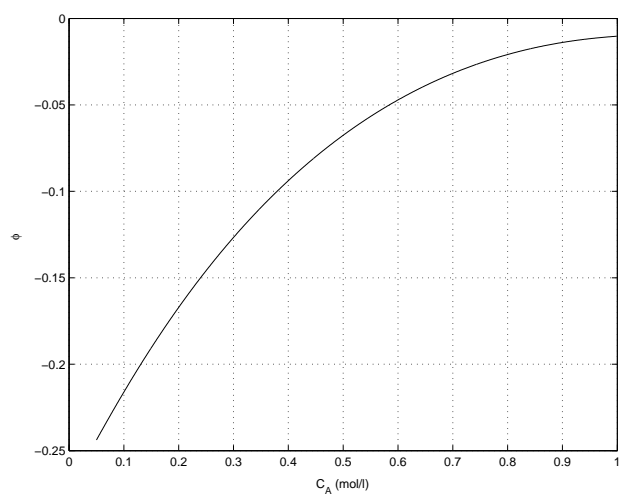

Fig. 14. Entropy flux $\left(\phi_{s}\right)$ for the reactor Fig. 15. Entropy production $\left(P_{s}\right)$ for the with PI control loop for $T$

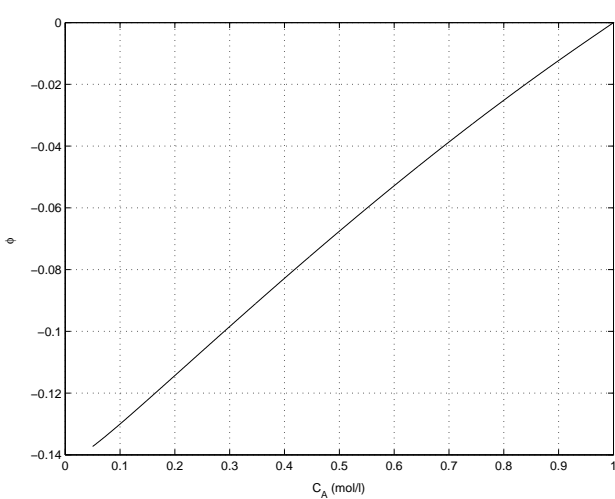

Fig. 13. Entropy flux $\left(\phi_{s}\right)$ for the open loop reactor

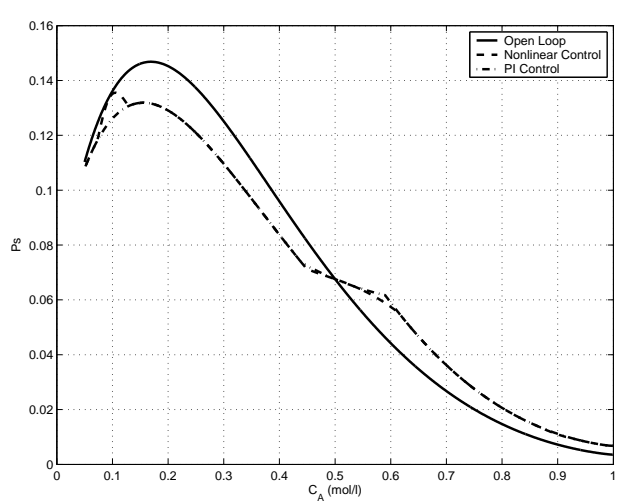

open and closed (PI and nonlinear control of $T$ ) loop reactor

The proper performance of this intensive PI control is confirmed by representing the dynamic trajectories in the state space (Figure 16), where the convergence of all trajectories to a unique steady state point can be checked.

2. Nonlinear Control: We apply global linearization control for SISO systems (Isidori, 1995). The proposed feedback control law has the form:

$$
\bar{u}=\frac{-L_{f}^{2} h(\bar{x})-k_{0} \bar{x}_{1}-k_{1} L_{f} h(\bar{x})}{L_{g} L_{f}^{2} h(\bar{x})}
$$

where $x$ and $u$ are in deviation form and the parameters $k_{0}$ and $k_{1}$ are the 


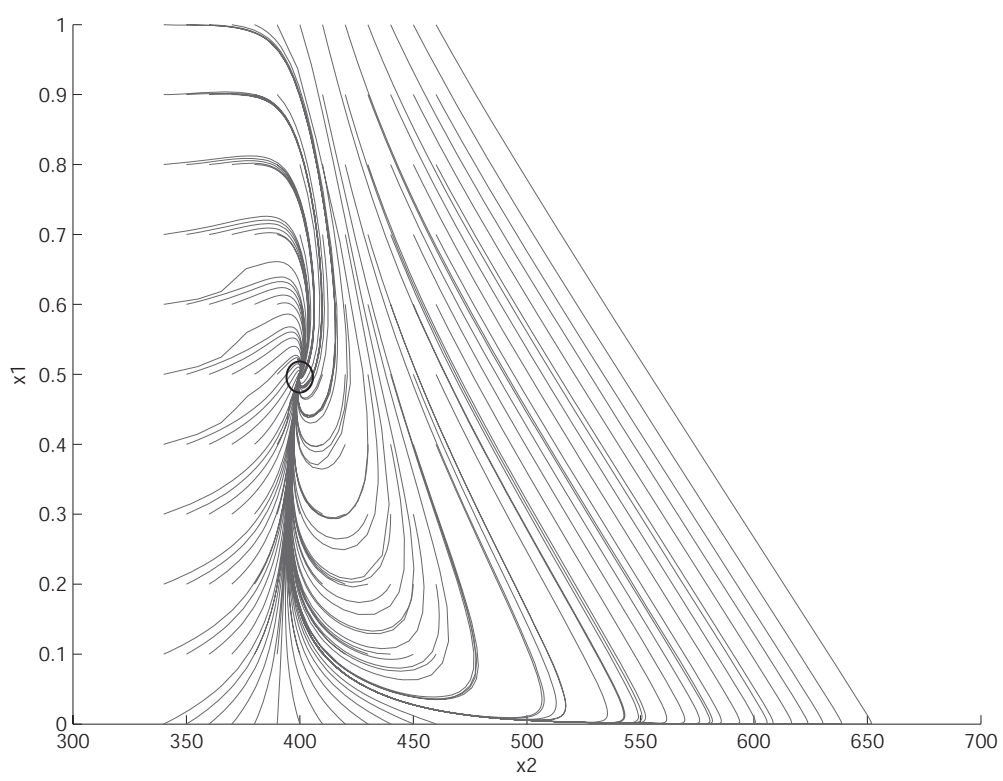

Fig. 16. State Space for the Non-Isothermal CSTR with PI control over $T$ gains of the controller. The Lie derivatives in (70) for this case are:

$$
\begin{aligned}
& L_{f} h(\bar{x})=\frac{\partial h}{\partial \bar{x}} f(\bar{x})=\left[\begin{array}{ll}
1 & 0
\end{array}\right]\left[\begin{array}{c}
\rho^{*}-\rho-\theta \bar{x}_{1} \\
\beta\left(\rho-\rho^{*}\right)-(\theta+\gamma) \bar{x}_{2}
\end{array}\right]=\rho^{*}-\rho-\theta \bar{x}_{1} \\
& L_{f}^{2} h(\bar{x})=\frac{\partial L_{f} h}{\partial \bar{x}} f(\bar{x})=\left[\begin{array}{ll}
-r-\theta-q \rho
\end{array}\right]\left[\begin{array}{c}
\rho^{*}-\rho-\theta \bar{x}_{1} \\
\beta\left(\rho-\rho^{*}\right)-(\theta+\gamma) \bar{x}_{2}
\end{array}\right] \\
& L_{g} L_{f} h=\frac{\partial L_{f} h}{\partial \bar{x}} g(\bar{x})=q \rho \gamma
\end{aligned}
$$

where $r$ and $q$ are:

$$
r=\frac{\partial \rho}{\partial \bar{x}_{1}}=e^{a-\frac{b}{\bar{x}_{2}+x_{2}^{*}}} ; \quad q=\frac{\partial \rho}{\partial \bar{x}_{2}}=\frac{b}{\left(\bar{x}_{2}+x_{2}^{*}\right)^{2}}\left(\bar{x}_{1}+x_{1}^{*}\right) e^{a-\frac{b}{\bar{x}_{2}+x_{2}^{*}}}
$$

By using the nonlinear control law presented in Eqn. (70) for $T_{c}$ with the gain values presented in the work of Alonso and Banga (1998) $\left(k_{0}=0.1054\right.$ and $k_{1}$ $=0.6581$ ), the entropy variation takes the form presented in Figure 17. As for the PI control case, there only exists a point where $d S=0$ due to changes in the entropy flux (Figure 18) caused by the nonlinear control. Note that there is another point very close to $d S=0$ but, as shown in Figure (17), it does not reach this value. This fact indicates that the considered nonlinear control is suitable to stabilize the system states in the desired set point.

The new space state for the closed loop system is depicted in Figure 19. The unique steady state corresponds to the chosen reference, where all the trajectories will converge to. 

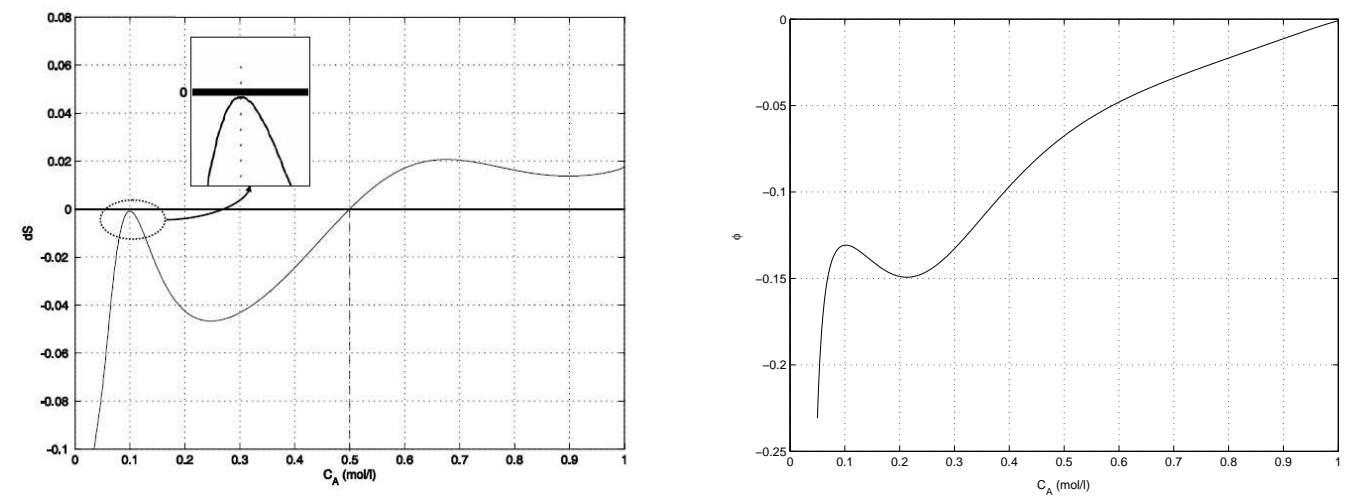

Fig. 17. Entropy variation $(d S)$ for the re- Fig. 18. Entropy flux $\left(\phi_{s}\right)$ for the reactor actor with a nonlinear control loop for $T$ with nonlinear control over $T$

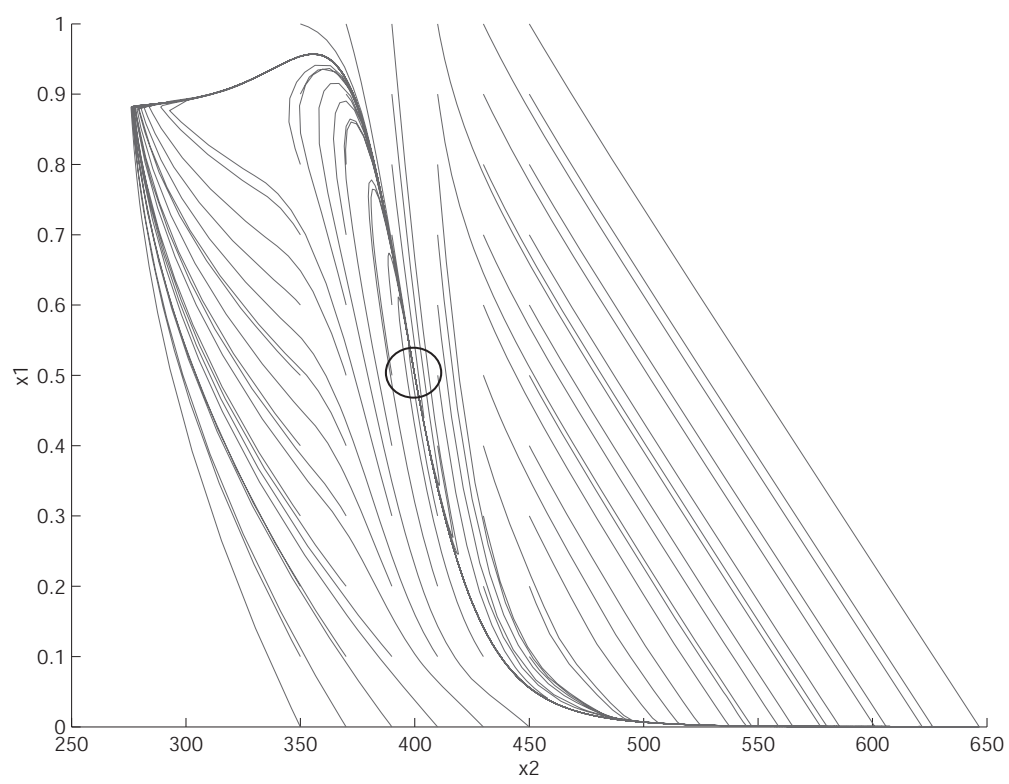

Fig. 19. State space for the non-isothermal CSTR with nonlinear control of $T$

Finally, some control experiments were carried out in order to test the unstabilizing effect of input constraints on the intensive variables closed loop dynamics. To that purpose, we assume a constraint in the manipulated variable $T_{c}$. In this particular case, the variation range will be set to:

$$
335 \leq T_{c} \leq 365
$$

For the constrained PI control, we can corroborate the existence of two stable steady states as shown in Figure 20. For the more interesting case corresponding to the constrained nonlinear control, it is possible to identify two stable steady states and a region where a limit cycle exists (Figure 21). This oscillatory behavior is generated by the fluctuation of the energy inventory that defines the convex region due to the control variable $T_{c}$ reaching upper and lower constraints. This can be shown by assuming an initial 


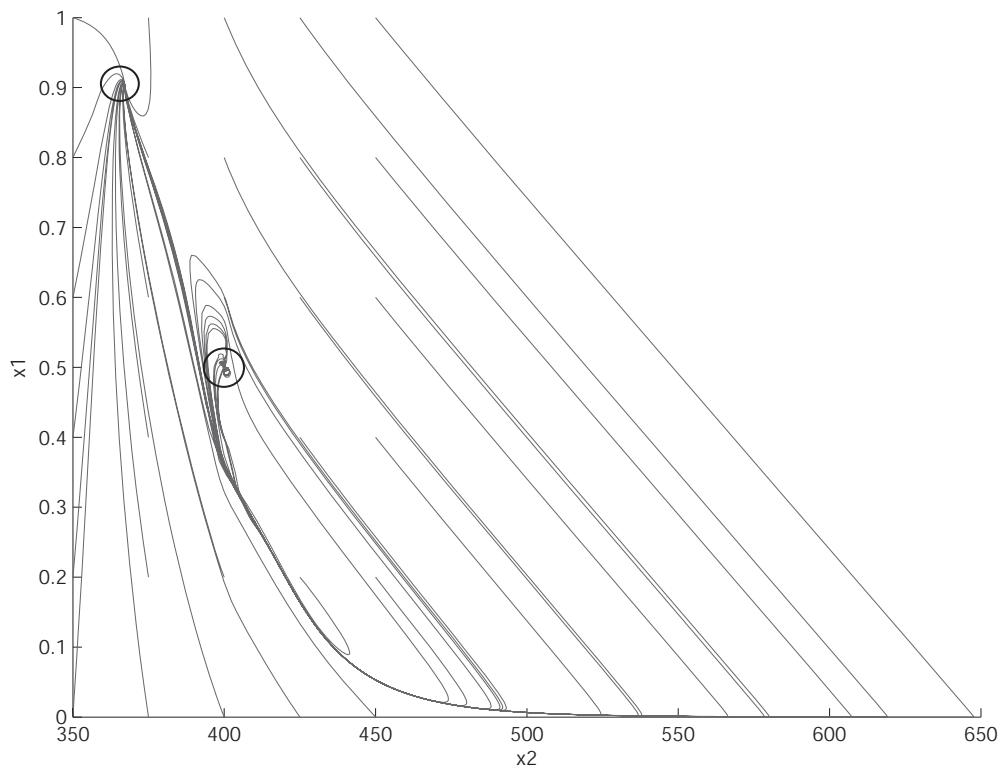

Fig. 20. State space for the non-isothermal CSTR with PI control of $T$ and $T_{c}$ bounded

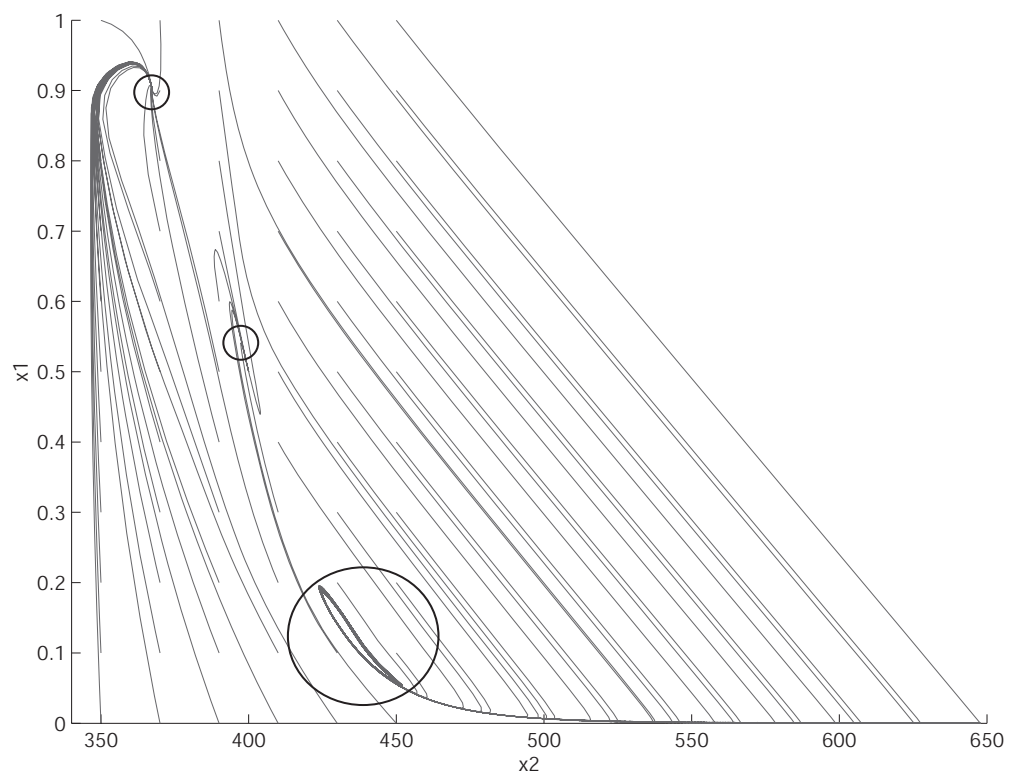

Fig. 21. State space for the non-isothermal CSTR with nonlinear control of $T$ and $T_{c}$ bounded

condition for the reactor near the limit cycle region $\left(C_{A}=0.25 \mathrm{~mol} / \mathrm{l}\right.$ and $T=420 \mathrm{~K}$ ). The evolution of the system to the limit cycle is presented in Figure 22. The fluctuation causes the energy inventory in the network to vary along time which, in turn, produces oscillations of the compact region respect to the nominal position. In this case, the thermodynamic analysis reveals new points in the region $\Lambda$ satisfying the stationary entropy balance (see Figure 23). These corresponds with either stable, unstable or even limit cycles caused 

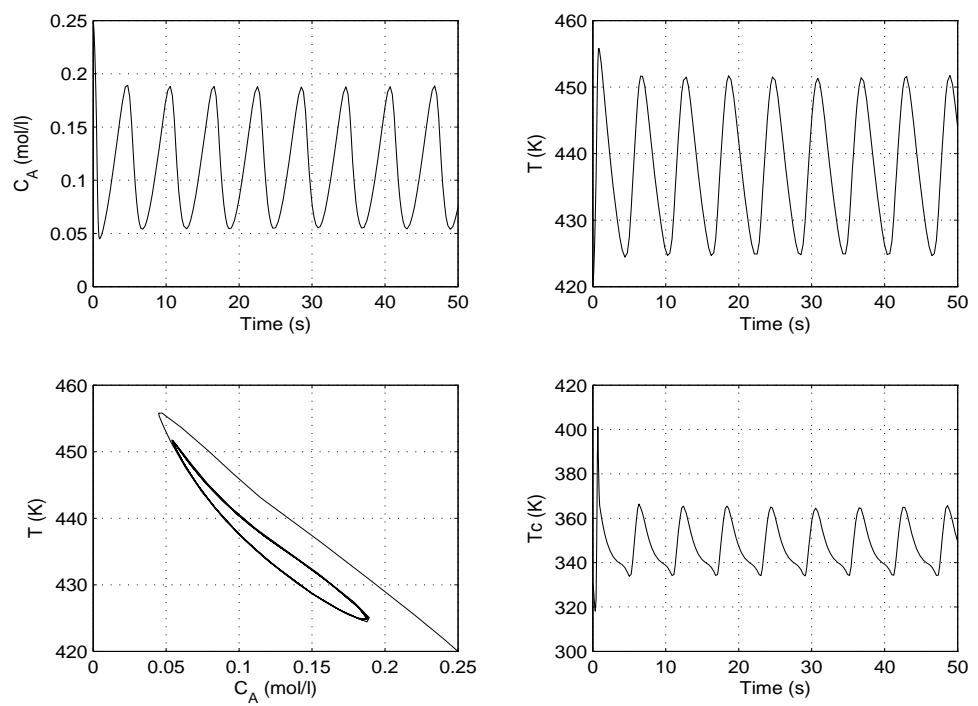

Fig. 22. Evolution of the intensive variables $\left(C_{A}\right.$ and $\left.T\right)$ and manipulated variable $\left(T_{c}\right)$ in the presence of input constraints for the nonlinear control of $T$

by total inventory fluctuations.

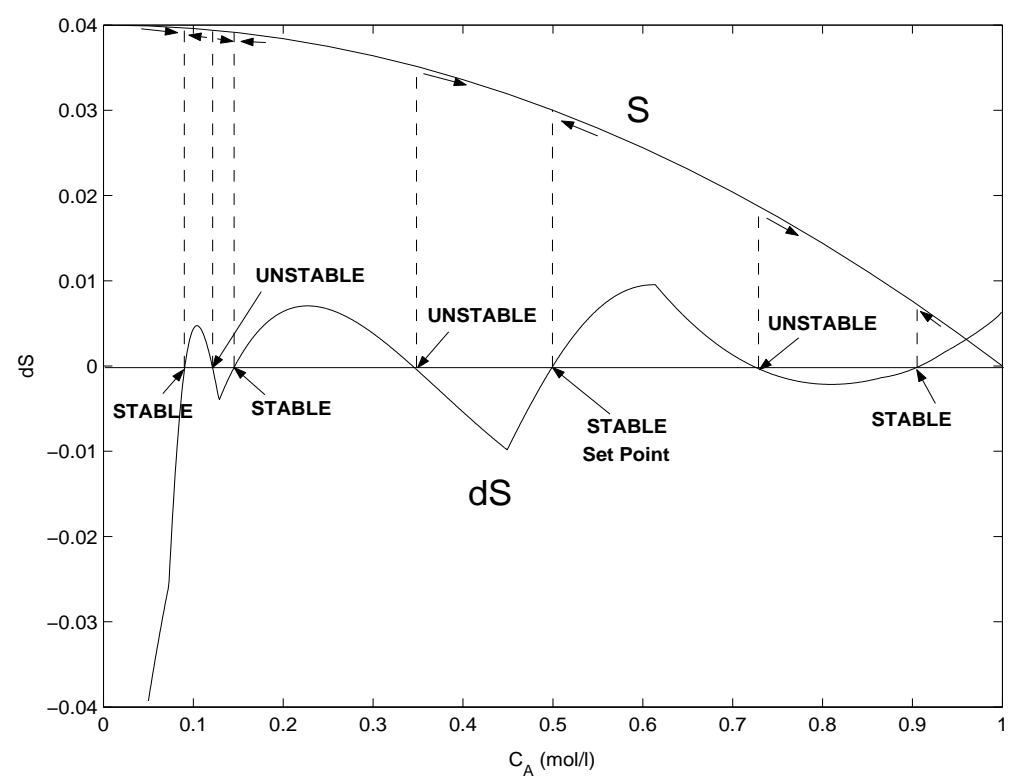

Fig. 23. Reactor steady states stability analysis in the presence of input constraints for the nonlinear control of $T$.

\section{Conclusions}

In this paper, a systematic plant-wide control design methodology has been presented. The approach, which combines tools and concepts from systems the- 
ory and thermodynamics, allows the design of decentralized control structures which simultaneously ensure stabilization of both plant extensive and intensive variables: inventory control loops are designed first to guarantee that the states of the plant will remain on a convex invariant region. Intensive variable control loops are then designed to avoid multiplicities and ensure the appropriate convergence rate. The proposed methodology has been illustrated on a reactor network exhibiting complex behavior, achieving the stabilization of the system states in the case of unconstrained control of the intensive variables. For the constrained input case, complex behavior cannot be handled properly. The dynamical results were also confirmed using the entropy balance, which is revealed as a powerful tool to analyze the stability or unstability of the steady states of the system.

\section{Acknowledgements}

The authors acknowledge the financial support received from the Spanish Government (MCyT Projects PPQ2001-3643), Xunta de Galicia (PGIDIT02PXIC40209PN) and "PRIMS" Marie Curie Action (MRTN-CT-2004-512233).

\section{References}

Alonso, A. A., Banga, J. R., 1998. Design of a class of stabilizing nonlinear state feedback controllers with bounded inputs. Ind. Eng. Chem. 37, 141144.

Alonso, A. A., Ydstie, B. E., 1996. Process systems, passivity and the second law of thermodynamics. Comp. \& Chem. Eng. 20, S1119-S1124.

Alonso, A. A., Ydstie, B. E., 2001. Stabilization of distributed systems using irreversible thermodynamics. Automatica 37 (11), 1739-1755.

Alvarez, J., Alvarez, J., Suarez, R., 1991. Nonlinear bounded control for a class of continuous agitated reactors. Chemical Engineering Science 46, 32353249 .

Antelo, L., Ydstie, B., Alonso, A., 2005. A thermodynamic approach to to the stability of equilibrium mass transfer units and multi-stage distillation columns. Proceedings of the 2005 AIChE Annual Meeting .

Bao, J., Zhang, W. Z., Lee, P. L., 2002. Passivity-based decentralized failuretolerant control. Ind. Eng. Chem. 41, 5702-5715.

Biegler, L., Grossman, I., 2004. Retrospective on optimization. Comp. and Chem. Eng. 28 (8), 1169-1192.

Buckley, P., 1964. Techniques of Process Control. John Wiley \& Sons.

Callen, H., 1985. Thermodynamics and an Introduction to Thermostatistics. 2nd. Edition. John Wiley \& Sons. 
Cordonier, G., Schmidt, L., Aris, R., 1990. Forced oscillations of chemical reactors with multiple steady states. Chemical Engineering Science 45, 16591675.

Farschman, C., Viswanath, K., Ydstie, B., 1998. Process systems and inventory control. AIChE Journal 44 (8), 1841-1857.

Gonzalez, P., Alvarez, J., 2005. Combined proportional/integral inventory control of solution homopolymerization reactors. Ind. Eng. Chem. 44, 71477163.

Hangos, K. M., Alonso, A. A., Perkins, J. D., Ydstie, B. E., 1999. Thermodynamic approach to the structural stability of process plants. AIChE Journal $45(4), 802$.

Isidori, A., 1995. Nonlinear Control Systems. Springer-Verlag, Berlin.

Jillson, K., Ydstie, B., 2005. Complex process networks: passivity and optimality. Proceedings of the 16th IFAC World Congress ISBN: 0-08-045108-X.

Keenan, J., 1951. Availability and irreversibility in thermodynamics. British Journal of Applied Physics 2, 183.

Kevrekidis, I. G., Aris, R., Schmidt, L. D., 1986. The stirred tank forced. Chemical Engineering Science 41, 1549-1560.

Larsson, T., Skogestad, S., 2000. Plantwide control: A review and a new design procedure. Modelling, Identification and Control 21, 209-240.

Luyben, M., Tyreus, B., Luyben, W., 1997. Plantwide control design procedure. AIChE Journal 43 (12), 3161-3174.

Ruszkowski, M., Garcia-Osorio, V., Ydstie, B., 2005. Passivity based control of transport reactor systems. AIChE Journal 51 (12), 3147-31266.

Skogestad, S., 2002. Plantwide control: Towards a systematic procedure. In CAPE 10 and ESCAPE $12,57-69$.

Slotine, J., Li, W., 1991. Applied Nonlinear Control. Prentice Hall, Englewood Cliffs, New Jersey.

Van der Shaft, A., 2000. $L_{2}$-Gain and Passivity Techniques in Nonlinear Control. Springer.

Ydstie, B. E., Alonso, A. A., 1997. Process systems and passivity via the clausius-plank inequality. Syst. \& Cont. Letters 30 (5), 253-264. 